\title{
RELATIVE TWISTING IN OUTER SPACE
}

\author{
MATT CLAY AND ALEXANDRA PETTET
}

\begin{abstract}
Subsurface projection is indispensable to studying the geometry of the mapping class group and the curve complex of a surface. When the subsurface is an annulus, this projection is sometimes called relative twisting. We give two alternate versions of relative twisting for the outer automorphism group of a free group. We use this to describe sufficient conditions for when a folding path enters the thin part of Culler-Vogtmann's Outer space. As an application of our condition, we produce a sequence of fully irreducible outer automorphisms whose axes in Outer space travel through graphs with arbitrarily short cycles; we also describe the asymptotic behavior of their translation lengths.
\end{abstract}

\section{INTRODUCTION}

Culler and Vogtmann gave the first account of Outer space $C V_{k}$ in their 1986 paper [20]: elements are finite marked projectivized metric graphs with fundamental group $F_{k}$, the rank $k$ non-abelian free group, and two graphs are close when the lengths of some finite collection of elements of $F_{k}$ are close. By considering the universal covers of the marked graphs, $C V_{k}$ is also described as the space of free simplicial minimal isometric actions of $F_{k}$ on $\mathbb{R}$-trees. Topologically, $C V_{k}$ has the structure of a contractible simplicial complex (missing some faces) on which Out $F_{k}$ acts properly and simplicially by changing markings. Metrically, however, Outer space remains largely a mystery. Much of the conjectural picture for Outer space geometry comes from Teichmüller theory, where the Teichmüller metric, the Weil-Petersson metric, and the Thurston metric have been defined and extensively studied. Unfortunately Outer space lacks much of the structure that paves the way for these metrics; perhaps most notably, $C V_{k}$ is not a manifold.

Of the three metrics on Teichmüller space mentioned above, only the third, the Thurston metric, has been interpreted in the Outer space setting; there it is more commonly referred to as the Lipschitz metric.

The first author is partially supported by NSF grant DMS-1006898. The second author was partially supported by NSF grant DMS-0856143, NSF RTG DMS-0602191, EPSRC grant EP/D073626/2, and an NSERC Discovery Grant. 
Features of this metric were recorded by Francaviglia-Martino in [22, 23].

Algom-Kfir (see also Hamenstädt [26]) proved that axes of fully irreducible elements of Out $F_{k}$ are strongly contracting, so that $C V_{k}$ exhibits a characteristic of negative curvature in these directions. Her result was anticipated by a theorem of Minsky [36], which showed that Teichmüller geodesics contained in the $\epsilon$-thick part of Teichmüller space are strongly contracting, uniformly depending on $\epsilon$. Algom-Kfir's contraction constants depend on the outer automorphisms to which they belong. The question of whether these constants only depend on the geometry of the graphs along the axes has not been addressed.

For $\epsilon>0$, we define $C V_{k}^{\epsilon}$ as the subset of $C V_{k}$ consisting of graphs that contain a cycle of length less than $\epsilon$. We should perhaps resist calling $C V_{k}^{\epsilon}$ the "thin part" of Outer space as it is not clear that AlgomKfir's theorem extends uniformly to geodesics in the complement of $C V_{k}^{\epsilon}$. Nevertheless, this set does hold some nice properties analogous to those of the thin part of Teichmüller space; for instance, the cusps of the quotient $C V_{k} /$ Out $F_{k}$ are contained in $C V_{k}^{\epsilon}$, and the quotient $\left(C V_{k}-C V_{k}^{\epsilon}\right) /$ Out $F_{k}$ of the complement is quasi-isometric to Out $F_{k}$.

The main results of this paper provide conditions, akin to those of Rafi [37] in the setting of Teichmüller space, that guarantee that a geodesic or an axis of a fully irreducible element travel through $C V_{k}^{\epsilon}$. Our criteria are based on a notion of relative twisting in Outer space. We come at this from two different points of view, each motivated by the quest to find satisfactory analogues of subsurface projection and relative twisting from the theory of mapping class groups [35, 21].

Geometric: Our first approach to relative twisting directly adapts the original geometric definition to free groups. We give a pairing $\tau_{a}\left(G, G^{\prime}\right)$ between two graphs $G, G^{\prime} \in C V_{k}$ relative to some nontrivial $a \in F_{k}$, which we define by means of the Guirardel core [24]. This is a certain 2-complex associated to the graphs that provides a means of selecting a geometry for $F_{k}$ that "sees" both $G$ and $G^{\prime}$. We obtain a condition on the graphs that, when satisfied, enables us to construct a connecting geodesic between them, traveling through $C V_{k}^{\epsilon}$.

Theorem 5.2. Suppose $G, G^{\prime} \in C V_{k}$ with $d=d_{L}\left(G, G^{\prime}\right)$ such that $\tau_{a}\left(G, G^{\prime}\right) \geq n+2$ for some $a \in F_{k}$. Then there is a geodesic $\alpha:[0, d] \rightarrow$ $C V_{k}$ such that $\alpha(0)=G$ and $\alpha(d)=G^{\prime}$ and for some $t \in[0, d]$, we have $\ell_{\alpha(t)}(a) \leq 1 / n$. In other words, $\alpha([0, d]) \cap C V_{k}^{1 / n} \neq \emptyset$.

As a corollary, we get the following lower bound the distance between two marked graphs in $C V_{k}$. 
Corollary 1.1. Suppose $G, G^{\prime} \in C V_{k}$ and $G^{\prime}$ does not have a cycle of length less than $\epsilon$. Then:

$$
d_{L}\left(G, G^{\prime}\right) \geq \log \sup _{1 \neq a \in F_{k}} \epsilon \tau_{a}\left(G, G^{\prime}\right)
$$

Proof. Let $a \in F_{k}$ be nontrivial. If $\tau_{a}\left(G, G^{\prime}\right) \geq n$, then by Theorem 5.2 , there is a geodesic $\alpha:[0, d] \rightarrow C V_{k}$ such that $\alpha(0)=G$ and $\alpha(d)=G^{\prime}$, and for some $t \in[0, d]$ that $\ell_{\alpha(t)}(a) \leq 1 / n$. As $G^{\prime}$ does not have a cycle of length less than $\epsilon$, it will follow from Proposition 2.1 that $d_{L}\left(\alpha(t), G^{\prime}\right) \geq \frac{\epsilon}{1 / n}=\epsilon n$. As $\alpha(t)$ is on a geodesic from $G$ to $G^{\prime}$, the corollary holds.

The similar lower bound for Teichmüller space is a special case of a theorem of Rafi [38].

Algebraic: The second point of view to relative twisting gives a pairing $\tau_{a}(T, \Lambda)$ between a tree $T \in \overline{C V}_{k}$ and an algebraic lamination $\Lambda$ of $F_{k}$ relative to some nontrivial $a \in F_{k}$. This pairing measures how the axes of $a$ in $T$ overlap with the leaves of the lamination. It is similar to the notion of "twisting" used by Alibegovic [3]. We obtain a criterion that implies that the axis of a fully irreducible element travels through $C V_{k}^{\epsilon}$ in terms of its unstable tree and lamination.

Theorem 5.3. Suppose $\phi \in$ Out $F_{k}$ is fully irreducible, with unstable tree $T_{-}$and lamination $\Lambda_{-}$such that $\tau_{a}\left(T_{-}, \Lambda_{-}\right) \geq n+4$ for some $a \in F_{k}$. Then given any train-track $G$, there is an axis $\mathcal{L}_{\phi}$ for $\phi$ that contains $G$ and a graph $G_{0}$ with $\ell_{G_{0}}(a) \leq 1 / n$. In other words, $\mathcal{L}_{\phi} \cap C V_{k}^{1 / n} \neq \emptyset$.

As an application of Theorem 5.3, we examine outer automorphisms of $F_{k}$ that are products of powers of two Dehn twists $\delta_{1}$ and $\delta_{2}$ which "fill" in an appropriate sense. We show (Section 6) that axes for $\delta_{1}^{n} \delta_{2}^{-n}$ travel through graphs with a cycle of length $\sim 1 / n$. Moreover, we can estimate their translation lengths on $C V_{k}$; we compute that they grow logarithmically in $n$ (Theorem 6.6).

The proofs of Theorems 5.2 and 5.3 are similar. In both cases we show that large relative twist implies the existence of a certain path that contains a large power of $a$ (Propositions 3.5 and 4.8). These paths, called vanishing paths, are folded, either in the map $G \rightarrow G^{\prime}$ or in a train-track map representing $\phi$, and are homotopically trivial in the image. The most efficient way to fold over a loop representing a several times is to first make $a$ a short loop (Proposition 5.1).

It appears likely that our definition of algebraic twist (at least as used in Theorem 5.3) is a special case of our definition of geometric twist. We anticipate investigating this relationship in a further paper. 
The paper is organized as follows. In Section 2 we review some of the basic theory of Outer space and the Lipschitz metric, irreducible outer automorphisms, train-track maps, and laminations; only Section 2.5 contains some new material. As this section is already lengthy, some background, such as a summary of currents for free groups, is suppressed until it is needed in Section 6. In Section 3, following an outline of some properties of Guirardel's core and a brief review of relative twisting for the mapping class group, the first, "geometric," analogue of relative twisting for Out $F_{k}$ is given. Section 4 is concerned with the second, "algebraic," notion of relative twisting. Each of Sections 3 and 4 conclude with a proposition essential to the proofs of the main theorems, found in Section 5. In Section 6, we bring together results from Section 5 and previous papers of the authors [12, 13] to describe a method for constructing geodesic axes of fully irreducible elements which enter the thin part of Outer space.

Acknowledgments The authors would like to thank Kasra Rafi and Juan Souto for helpful conversations concerning this project. Additionally, the authors thank the referee for a careful reading of this work with helpful suggestions.

\section{PRELiminaries}

2.1. Outer space. We begin by fixing a generating set of the free group $F_{k}=\left\langle x_{1}, \ldots, x_{k}\right\rangle$. Let $G$ be a simplicial graph, i.e., a onedimensional cell complex, with $\pi_{1}(G)$ isomorphic to $F_{k}$. Let $R$ be a wedge of $k$ (oriented) circles, with each circle identified to one of the generators of $F_{k}$. Then by a marking of $G$ we mean a homotopy equivalence $\rho: R \rightarrow G$. From the map $\rho_{*}: \pi_{1}(R) \rightarrow \pi_{1}(G)$, we then have an identification of $F_{k}$ with $\pi_{1}(G)$. Given two marked metric graphs $\rho_{1}: R \rightarrow G_{1}$ and $\rho_{2}: R \rightarrow G_{2}$, a map $f: G_{1} \rightarrow G_{2}$ is a change of marking if it is linear on edges, and if $f \circ \rho_{1}: R \rightarrow G_{2}$ is homotopic to $\rho_{2}: R \rightarrow G_{2}$. A topological representative of $\phi \in$ Out $F_{k}$ is a marked graph $\rho: R \rightarrow G$, together with a self homotopy equivalence $g: G \rightarrow G$, so that the homotopy equivalence $\rho^{-1} \circ g \circ \rho: R \rightarrow R$ induces $\phi$ on $\pi_{1}(R)=F_{k}$.

We denote by $c v_{k}$ the unprojectivized (Culler-Vogtmann) Outer space consisting of marked metric graphs $G$, where $\pi_{1}(G)=F_{k}$ and the degree of every vertex of $G$ is at least 3 . Two points $\rho_{1}: R \rightarrow G_{1}$ and $\rho_{2}: R \rightarrow G_{2}$ in $c v_{k}$ are equivalent if there is an isometry $\iota: G_{1} \rightarrow G_{2}$ so that $\iota \circ \rho_{1}$ is homotopic to $\rho_{2}$. An alternate description of $c v_{k}$ is as the space of free minimal isometric $F_{k}$-actions on simplicial trees, and we will alternate freely between treating Outer space as a space of 
trees and as a space of graphs. There is a right action of Out $F_{k}$ given by precomposing the marking (or $F_{k}$-action) by a representative of the outer automorphism. Outer space is defined as the projectivization of $c v_{k}: C V_{k}=c v_{k} / \mathbb{R}_{>0}$; it can be identified with the subspace of $c v_{k}$ consisting of marked graphs whose edge lengths sum to 1.

To simplify the notation for elements in Outer space, we denote a marked metric graph $\rho: R \rightarrow G$ simply by $G$. A path in $G$ is a continuous map $\alpha: I \rightarrow G$, where $I$ is an interval of $\mathbb{R}$. For convenience, and when it is clear from context, $\alpha$ may denote either the map or its image in $G$; while $[\alpha]$ will denote the image of $\alpha$ after "pulling it tight," i.e., the image of any immersed homotopy (relative endpoints) representative of $\alpha$. We then use $L_{G}(\alpha)$ to denote the length of $[\alpha]$ in $G$. For an element $a \in F_{k}$, we write $\ell_{G}(a)$ to denote the minimal length of a loop in $G$ representing the conjugacy class of $a$.

For points $x, y \in T$, we use $[x, y]$ to denote the image of the unique tight path connecting $x$ and $y$ in $T$. For an $F_{k}$-tree $T$ and an element $a \in F_{k}$, we write $\ell_{T}(a)$ to denote the minimal translation length of $a$ in $T$. If $\ell_{T}(a) \neq 0$, then $a$ has an invariant axis $T^{\langle a\rangle}$ and $\operatorname{vol}\left(T^{\langle a\rangle} /\langle a\rangle\right)=$ $\ell_{T}(a)$. If $G$ is a graph with fundamental group $F_{k}$, then $\widetilde{G}$ denotes the universal cover of $G$, with a chosen base point so that there is an $F_{k}$-action on $\widetilde{G}$. Clearly $\ell_{G}=\ell_{\widetilde{G}}$.

Using the description as a space of tree actions, $c v_{k}$ is topologized via the the axes topology. That is, a tree $T \in c v_{k}$ is identified with a point in $\mathbb{R}^{F_{k}}$ by the coordinates $\left(\ell_{T}(g)\right)_{g \in F_{k}}[19]$. Cohen and Lustig proved that the space of very small actions on $\mathbb{R}$-trees contains the closure $\overline{c v}_{k}$ of $c v_{k}$ [15]. The converse, that every very small minimal action on an $\mathbb{R}$-tree is the limit of free minimal simplicial actions, was shown by Bestvina and Feighn [6]. Recall that an action of $F_{k}$ on an $\mathbb{R}$-tree is very small if arc stabilizers are trivial or maximal cyclic, and the stabilizer of any tripod is trivial.

Given two points $G_{1}$ and $G_{2}$ in the projectivized Outer space $C V_{k}$, let $f: G_{1} \rightarrow G_{2}$ be a change of marking, and denote by $\sigma(f)$ the maximal slope of $f$ (recall that $f$ is linear on edges). We have the following proposition, due to White (see $[1,5]$ ):

Proposition 2.1 (White). Let $G_{1}, G_{2}$ be two graphs in $C V_{k}$. Then:

$$
\inf \left\{\sigma(f) \mid f: G_{1} \rightarrow G_{2} \text { change of marking }\right\}=\sup _{1 \neq a \in F_{k}} \frac{\ell_{\widetilde{G}_{2}}(a)}{\ell_{\widetilde{G}_{1}}(a)}
$$

Moreover both inf and sup are realized. 
For $G_{1}$ and $G_{2}$ in Out $F_{k}$, let $\sigma\left(G_{1}, G_{2}\right)$ be the value in Proposition 2.1. We define a function $d_{L}: C V_{k} \times C V_{k} \rightarrow \mathbb{R}_{\geq 0}$ by

$$
d_{L}\left(G_{1}, G_{2}\right)=\log \sigma\left(G_{1}, G_{2}\right) .
$$

Its only failure to be a distance is that it is not symmetric; it is not hard to construct examples of $G_{1}, G_{2} \in C V_{k}$ with $d_{L}\left(G_{1}, G_{2}\right) \neq d_{L}\left(G_{2}, G_{1}\right)$ (see [2]). In spite of this anomaly, we will refer to $d_{L}$ as the Lipschitz metric on $C V_{k}$. We remark that it is known that the minimal Lipschitz constant, taken over all continuous maps $f: G_{1} \rightarrow G_{2}$ such that $f \circ \rho_{1}$ is homotopic to $\rho_{2}$, is achieved by a map that is linear on edges $([1,23])$.

Example 2.2. We present an example of computing distances in $C V_{k}$ that will be relevant to those examples constructed in Section 6. Fix a basis $\mathcal{T}=\mathcal{A} \cup\{t\}$ of $F_{k}$ and an element $c \in\langle\mathcal{A}\rangle$ that is cyclically reduced with respect to $\mathcal{T}$. Consider the Cayley tree $T$ and the marked graph $G=T / F_{k}$, metrized so that all edge lengths are equal to $1 / k$.

Let $\delta$ be the automorphism that sends $t$ to $c t$ and acts as the identity on $\langle\mathcal{A}\rangle$. Then there is a change of marking map $f: G \rightarrow G \delta^{n}$ defined by subdividing the edge corresponding to $t$ into $n+1$ edges and sending each of the first $n$ edges over the edge path for $c$ and the last edge over the edge corresponding to $t$. Therefore, the image of the edge $t$ has length:

$$
n \ell_{G}(c)+\frac{1}{k}=\frac{n k \ell_{G}(c)+1}{k}
$$

and hence the edge $t$ has been stretched by $n k \ell_{G}(c)+1$. Since the edge corresponding to $t$ is the only edge stretched and since it is mapped to a tight loop we have that:

$$
d_{L}\left(G, G \delta^{n}\right)=\log \left(n k \ell_{G}(c)+1\right) .
$$

In the terminology from the proof of Theorem 2.1 in [1], the loop $t$ is the subgraph $G_{f} \subset G$ and it is a legal loop.

The automorphism $\delta$ is an example of a Dehn twist automorphism (see Section 6); in this case corresponding to the Bass-Serre tree arising from the HNN-extension $\left\langle\mathcal{A}, c_{0}, t \mid t^{-1} c t=c_{0}\right\rangle$. We refer to such a tree as a cyclic tree. For the case of a cyclic tree dual to an amalgamated free product $\langle\mathcal{A}\rangle *\langle c\rangle\langle c, \mathcal{B}\rangle$ and its associated Dehn twist $\left(a \mapsto a, b \mapsto c b c^{-1}\right)$, one can also show, using similar methods, that the distance from $G$ to $G \delta^{n}$ is approximately $\log n$. In this case though, the obvious map sending edges corresponding to elements $b \in \mathcal{B}$ to $c^{n} b c^{-n}$ is not the optimal map. Instead one sends $b$ to $c^{n / 2} b c^{-n / 2}$ and edges corresponding to elements $a \in \mathcal{A}$ to $c^{-n / 2} a c^{n / 2}$.

We remark for use in Section 6, that $d_{L}\left(G \delta^{n}, G\right)=d_{L}\left(G, G \delta^{n}\right)$. Indeed, $d_{L}\left(G \delta^{n}, G\right)=d_{L}\left(G, G \delta^{-n}\right)$ and the same argument as above 
shows that $d_{L}\left(G, G \delta^{-n}\right)=\log \left(n k \ell_{G}\left(c^{-1}\right)+1\right)$. But of course $\ell_{G}(c)=$ $\ell_{G}\left(c^{-1}\right)$.

2.2. Bounded backtracking. Suppose that $f: T \rightarrow T^{\prime}$ is a continuous map, where $T$ and $T^{\prime}$ are trees. We say that $f$ has bounded backtracking if there is a constant $C$ such that for any path $[x, y] \subset T$ from $x$ to $y$ in $T$, and any $z \in[x, y]$, necessarily $d_{T^{\prime}}([f(x), f(y)], f(z)) \leq C$. We denote by $B B T(f)$ the minimal such constant $C$. We note that for any given $T \in c v_{k}$ and $T^{\prime} \in \overline{c v}_{k}$, that any $F_{k}$-equivariant map $f: T \rightarrow T^{\prime}$ has bounded backtracking. Moreover $B B T(f) \leq \operatorname{Lip}(f) \operatorname{vol}_{T}\left(T / F_{k}\right)$, where $\operatorname{Lip}(f)$ is the Lipschitz constant of the map $f[7]$. In particular, if $T$ and $T^{\prime}$ are contained in the projectivized space $C V_{k}$, then $B B T(f) \leq \operatorname{Lip}(f)$.

For a path $\alpha \subset T$, denote by $\alpha \dagger_{L}$ the path obtained by deleting the extremal paths of length $L$. The following is an easy consequence of bounded backtracking.

Lemma 2.3. Suppose that $T, T^{\prime} \in \overline{c v}_{k}$, that $f: T \rightarrow T^{\prime}$ is an $F_{k}-$ equivariant map that has bounded backtracking, and that $\ell: \mathbb{R} \rightarrow T$ is a parametrized geodesic. If $L>B B T(f)$, and if for some interval $I \subset \mathbb{R}$, a tight path $\alpha \subset T^{\prime}$ is contained in $[f(\ell(I))] \dagger_{L}$, then necessarily $\alpha \subset\left[f\left(\ell\left(I^{\prime}\right)\right)\right]$ for any interval $I^{\prime} \supset I$.

Proof. Let $I=[x, y] \subset \mathbb{R}$ and assume that the hypotheses of the lemma hold, so that $d_{T^{\prime}}(f(\ell(x)), \alpha) \geq L$ and $d_{T^{\prime}}(f(\ell(y)), \alpha) \geq L$. Next let $x^{\prime} \in I$ be such that $f\left(\ell\left(x^{\prime}\right)\right)$ is the endpoint of $\alpha$ closest to $f(\ell(x))$, and let $y^{\prime} \in I$ be such that $f\left(\ell\left(y^{\prime}\right)\right)$ is the endpoint of $\alpha$ closest to $f(\ell(y))$.

Now suppose that $\alpha \not \subset\left[f\left(\ell\left(I^{\prime}\right)\right)\right]$ for some interval $I^{\prime}$ that contains $I$. As $T^{\prime}$ is a tree, either there exists an $x^{\prime \prime} \in I^{\prime}-I$ such that $f\left(\ell\left(x^{\prime \prime}\right)\right)=f\left(\ell\left(x^{\prime}\right)\right)$ or there exists an $y^{\prime \prime} \in I^{\prime}-I$ such that $f\left(\ell\left(y^{\prime \prime}\right)\right)=f\left(\ell\left(y^{\prime}\right)\right)$; without loss of generality, we assume the former. Then the path $\left[\ell\left(x^{\prime}\right), \ell\left(x^{\prime \prime}\right)\right] \subset T$ and the point $\ell(x) \in\left[\ell\left(x^{\prime}\right), \ell\left(x^{\prime \prime}\right)\right]$ violate bounded backtracking, as

$$
d_{T^{\prime}}\left(\left[f\left(\ell\left(x^{\prime}\right), f\left(\ell\left(x^{\prime \prime}\right)\right], f(\ell(x))\right) \geq L>B B T(f) .\right.\right.
$$

2.3. Irreducible elements and train-tracks. Let $G$ be a graph. A turn is an unordered pair of oriented edges that share a common initial vertex. Letting $\bar{e}$ denote the edge $e$ with opposite orientation, we say that an edge path $\alpha$ crosses a turn $\left\{e_{1}, e_{2}\right\}$ if it contains an occurrence of either $\bar{e}_{1} e_{2}$ or $\bar{e}_{2} e_{1}$.

Let $g: G \rightarrow G$ be a homotopy equivalence that is linear on edges, mapping edges to edge paths. Then $g$ induces a map on the set of turns 
of $G$, as follows. Let $v$ be the common initial vertex of the edges of a turn $\left\{e_{1}, e_{2}\right\}$. Some initial segment of $e_{1}$ is mapped onto an edge $e_{1}^{\prime}$ based at $g(v)$, while some initial segment of $e_{2}$ onto an edge $e_{2}^{\prime}$, also based at $g(v)$. We assign $g\left(\left\{e_{1}, e_{2}\right\}\right)=\left\{e_{1}^{\prime}, e_{2}^{\prime}\right\}$.

The homotopy equivalence $g: G \rightarrow G$ is a train-track map if there is a collection $\mathcal{L} \mathcal{T}$ of turns such that:

(1) $\mathcal{L} \mathcal{T}$ is closed under iteration of $g$ and

(2) for an edge $e \subset G$, any turn crossed by $g(e)$ is in $\mathcal{L} \mathcal{T}$.

The unordered pairs of $\mathcal{L} \mathcal{T}$ are called legal turns, while an unordered pair of turns not in $\mathcal{L} \mathcal{T}$ is called an illegal turn. A path is legal if it only crosses legal turns. We will regularly refer to the underlying graph $G$ as a "train-track."

An element of $\phi \in$ Out $F_{k}$ is reducible if some conjugacy class of a proper free factor of $F_{k}$ is $\phi$-periodic; otherwise $\phi$ is irreducible. Bestvina and Handel proved that every irreducible element of Out $F_{k}$ has a topological representative that is a train-track map [8]. If $g: G \rightarrow$ $G$ is a train-track map representing an irreducible element of Out $F_{k}$, there is a metric on $G$ such that $g$ linearly expands each edge of $G$ by the same factor $\lambda$, called the expansion factor. This factor is the Perron-Frobenius eigenvalue of the transition matrix for $g$; a positive eigenvector for this eigenvalue specifies the metric on $G$.

All proper powers of a fully irreducible element $\phi$ of Out $F_{k}$ are irreducible. A fully irreducible element $\phi$ has the property that its minimal displacement in the Lipschitz metric is related to its expansion factor $\lambda_{\phi}$ by

$$
\min _{G \in C V_{k}} d_{L}(G, G \phi)=\log \left(\lambda_{\phi}\right) .
$$

Moreover, this minimum is realized by a train-track map for $\phi$. This relationship is one reason we choose not to symmetrize the Lipschitz metric, for typically the expansion factor of a fully irreducible element is not equal to that of its inverse. See for example [27].

For a fully irreducible element $\phi \in$ Out $F_{k}$ and any tree $T \in C V_{k}$, the sequence $T \phi^{n}$ has a well-defined limit $T_{+}(\phi)$ in $\overline{C V}_{k}$, called the stable tree of $\phi[7,33]$. The unstable tree for $\phi$, denoted by $T_{-}(\phi)$, is the stable tree for $\phi^{-1}$, i.e., $T_{-}(\phi)=T_{+}\left(\phi^{-1}\right)$. For an explicit description see [28]. We further note that if $T \in \overline{C V}_{k}-\left\{T_{-}(\phi)\right\}$, then $T \phi^{n}$ converges to $T_{+}(\phi)[33]$.

2.4. Geodesics in Outer space. Next, for an interval $I \subset \mathbb{R}$, we describe paths $I \rightarrow C V_{k}$ known as folding lines. We will be concerned with two types of such paths: those which connect two points $G_{1}$ and 
$G_{2}$ in the interior $C V_{k}$, and those which are axes of fully irreducible elements. The latter were studied by Algom-Kfir in [1].

For $G_{1}, G_{2} \in C V_{k}$, let $f: G_{1} \rightarrow G_{2}$ be a change of marking map whose Lipschitz constant realizes $\sigma\left(G_{1}, G_{2}\right)$. Find a path $\tilde{\alpha}_{1}$ based at $G_{1}$ contained in an open simplex of (unprojectivized) $c v_{k}$, along which edges of $G_{1}$ shrink just until the map induced by $f$ stretches every edge of the resulting graph by $\sigma\left(G_{1}, G_{2}\right)$; note that the lengths of those edges of $G_{1}$ that are stretched by exactly $\sigma\left(G_{1}, G_{2}\right)$ do not change along $\tilde{\alpha}_{1}$. Let the endpoint of the corresponding path $\alpha_{1}$ in (projectivized) $C V_{k}$ be $H_{1}$, with the change of marking map $h: H_{1} \rightarrow G_{2}$ induced by $f$. We choose a parameterization $\alpha_{1}:\left[0, d_{L}\left(G_{1}, H_{1}\right)\right] \rightarrow C V_{k}$ by arclength.

Now we construct a path $\alpha_{2}:\left[0, d_{L}\left(H_{1}, G_{2}\right)\right] \rightarrow C V_{k}$ with $\alpha_{2}(0)=H_{1}$ and $\alpha_{2}\left(d_{L}\left(H_{1}, G_{2}\right)\right)=G_{2}$. First subdivide the edges of $H_{1}$ to obtain a graph $H_{1}^{\prime}$ so that the preimage of vertices in $G_{2}$ consists of vertices in $H_{1}^{\prime}$, while the induced map $h: H_{1}^{\prime} \rightarrow G_{2}$ remains cellular. Select a vertex $v$ of $H_{1}^{\prime}$ at which two edges $e_{1}$ and $e_{2}$ identified by $h$ are based. Let $H(t)$ be the graph obtained from $H_{1}^{\prime}$ by folding the initial segments of length $\left(1-e^{-t}\right)$ of $e_{1}$ and $e_{2}$; let $\alpha_{2}(t)$ be the graph in $C V_{k}$ obtained from $H(t)$ by "forgetting" valence 2 vertices. Note that $d_{L}\left(H_{1}, \alpha_{2}(t)\right)=$ $t$. Define $\alpha_{2}(t)$ in this way until $e_{1}$ and $e_{2}$ are completely identified; then repeat the above with the resulting graph. Continue this process until the map induced by $f$ is an immersion in $G_{2}$; this is a finite process as $H_{1}^{\prime}$ has a finite number of vertices. Note that the immersion is necessarily an isometry as every edge is stretched by the same factor; thus the fold line just constructed connects $H_{1}$ to $G_{2}$ in $C V_{k}$. Finally, let $\alpha$ be the concatenation of $\alpha_{1}$ and $\alpha_{2}$; a path based at $G_{1}$ and terminating at $G_{2}$. Francaviglia and Martino [23, Theorem 5.5] proved that $\alpha:\left[0, d_{L}\left(G_{1}, G_{2}\right)\right] \rightarrow C V_{k}$ is a geodesic.

We can now describe a geodesic axis for a fully irreducible element $\phi$ of Out $F_{k}$. Let $\lambda_{\phi}$ be the expansion factor of $\phi$, and let $G$ be a train-track. To obtain a parametrized geodesic axis for $\phi$, first find a folding path $\alpha:\left[0, \log \left(\lambda_{\phi}\right)\right] \rightarrow C V_{k}$ as above, connecting $G$ to $G \phi$. Then define the graph $\alpha(t)=\alpha\left(t-n(t) \log \left(\lambda_{\phi}\right)\right) \phi^{n(t)}$, where $n(t)$ is the integer $\left\lfloor\frac{t}{\log \left(\lambda_{\phi}\right)}\right\rfloor$, and let $\mathcal{L}_{\phi}$ denote the image of $\alpha$. Algom-Kfir $[1$, Proposition 3.5] showed that $\alpha: \mathbb{R} \rightarrow C V_{k}$ is a geodesic parametrized by arclength.

2.5. Nielsen and vanishing paths. Suppose $G$ is a graph with a homotopy equivalence $g: G \rightarrow G$. We make note of two special types of paths in $G$ and collect some relevant results that will be useful for us in the sequel. 
First, a path $\alpha \subset G$ is a Nielsen path if $[g(\alpha)]=[\alpha]$; it is indivisible if it is not a concatenation of nontrivial Nielsen paths, so that any Nielsen path is a concatenation of indivisible Nielsen paths.

Theorem 2.4 ([28], Corollary 2.14). Suppose that $\phi \in$ Out $F_{k}$ is fully irreducible with stable tree $T_{+}=T_{+}(\phi)$, and that $g: G \rightarrow G$ is a traintrack representative. Then there is a surjective $F_{k}$-equivariant map $f_{g}: \widetilde{G} \rightarrow T_{+}$such that if $[x, y] \subset \widetilde{G}$ is a lift of a path $\alpha \subset G$ and $f_{g}(x)=f_{g}(y)$, then for some $m \geq 0$, the path $\left[g^{m}(\alpha)\right]$ is either a Nielsen path or trivial.

Definition 2.5. Let $a \in F_{k}$ and $T \in \overline{c v}_{k}$ be such that $\ell_{T}(a) \neq 0$. We say that a path $\alpha \subset T$ (possibly infinite) $n$-covers $a$ if $L_{T}\left(\alpha \cap T^{\langle a\rangle}\right) \geq$ $n \ell_{T}(a)$. In other words, $\alpha$ overlaps with a segment of the axis of $a$ for length at least $n \ell_{T}(a)$; there is a point $x \in \alpha$ such that $a^{n} x \in \alpha$ as well.

Similarly, given $G \in C V_{k}$, we say a path $\alpha \subset G n$-covers a if a lift of $\alpha$ to $\widetilde{G} n$-covers $a$. In other words, $\alpha$ decomposes into $\alpha=\beta \cdot \alpha_{0} \cdot \beta^{\prime}$, where $\alpha_{0}$ is the loop representing the conjugacy class of $a^{n}$.

Lemma 2.6. Let $\phi$ and $g: G \rightarrow G$ be as in Theorem 2.4. Suppose that a Nielsen path $\alpha$ in $G n$-covers $a$, for some $a \in F_{k}$ with $\ell_{T_{+}}(a)>0$ and $n \geq 2$. Then there is a subpath $\alpha_{0}$ of $\alpha$ which $n$-covers $a$ and which is contained in $\alpha^{\prime} \dagger_{\epsilon}$ for some indivisible Nielsen path $\alpha^{\prime}$ and $\epsilon>0$.

Proof. Suppose that $\alpha_{0}$ is a shortest subpath of $\alpha$ that $n$-covers $a$, but is not contained in the interior of an indivisible Nielsen path.

We can express $\alpha$ as a concatenation $\alpha_{1} \alpha_{2} \ldots \alpha_{r}$ of indivisible Nielsen paths $\alpha_{i}, i=1, \ldots, r$. The $g$-fixed points of $\alpha$ are precisely the endpoints of the $\alpha_{i}$ 's. Then since $\alpha_{0}$ is not contained in an indivisible Nielsen path, it must contain one of one of these fixed points $p$. It therefore contains at least $n$ copies of the fixed point $p$. Therefore some sequence $\alpha_{i} \ldots \alpha_{j}$ forms a Nielsen path and corresponds to the conjugacy class of $a$. A closed Nielsen path corresponds to a periodic loop. It follows that $\ell_{T_{+}}(a)=0$, contradicting the hypothesis on $a$.

A path $\alpha \subset G$ is a vanishing path of $g$ if $\left[g^{m}(\alpha)\right]$ is trivial (i.e., is a point) for some $m \geq 1$. We record the following observation from [4]:

Lemma 2.7. Let $\phi$ and $g: G \rightarrow G$ be as in Theorem 2.4, and suppose that $\alpha$ is an indivisible Nielsen path. Then any subpath $\beta \subseteq \alpha \dagger_{\epsilon}$ for some $\epsilon>0$ is contained in a vanishing path.

Proof. An indivisible Nielsen path can be decomposed into a sequence of legal paths as $\alpha=\alpha_{0} \cdot \beta_{0} \cdot \bar{\beta}_{1} \cdot \bar{\alpha}_{1}$, where $g\left(\alpha_{i}\right)=\alpha_{i} \cdot \beta_{i}$ and $g\left(\beta_{0}\right)=$ $g\left(\beta_{1}\right)$ [8]. Hence for $\epsilon>0$, with large enough $n$, the path $\left[g^{n}\left(\alpha \dagger_{\epsilon}\right)\right]$ is contained in $\beta_{0} \cdot \bar{\beta}_{1}$, and hence $\left[g^{n+1}\left(\alpha \dagger_{\epsilon}\right)\right]$ is trivial. 
Putting together Theorem 2.4 and Lemmas 2.6 and 2.7, we have the following:

Proposition 2.8. Suppose that $\phi \in$ Out $F_{k}$ is fully irreducible with stable tree $T_{+}=T_{+}(\phi)$, that $g: G \rightarrow G$ is a train-track representative of $\phi$, and that $a \in F_{k}$ is such that $\ell_{T_{+}}(a)>0$. Then there is a surjective $F_{k}$-equivariant map $f_{g}: \widetilde{G} \rightarrow T_{+}$such that if $[x, y] \subset \widetilde{G}$ is a lift of a reduced path $\alpha \subset G$ that $n$-covers $a$, for some $n \geq 2$, and if $f_{g}(x)=$ $f_{g}(y)$, then $\alpha$ contains a vanishing path that $n$-covers $a$.

2.6. Laminations and the map $\mathcal{Q}$. There are several notions of a "lamination" on a free group. For a full discussion on three different approaches and the relations between them, see $[16,17,18]$. We will only briefly describe the elements of the theory we need here.

The group $F_{k}$ is hyperbolic and hence has a boundary $\partial F_{k}$. We denote:

$$
\partial^{2} F_{k}=\left\{\left(x_{1}, x_{2}\right) \in \partial F_{k} \times \partial F_{k} \mid x_{1} \neq x_{2}\right\}
$$

This set is naturally identified with the space of oriented bi-infinite geodesics in a tree $T \in c v_{k}$ as we explain now.

An oriented bi-infinite geodesic is an isometric embedding $\ell: \mathbb{R} \rightarrow T$ considered up to reparametrization preserving the orientation. Any geodesic has two distinct endpoints in $\partial T$, denoted $\ell(\infty)$ and $\ell(-\infty)$. We can thus identify the geodesic $\ell$ with endpoints $(\ell(\infty), \ell(-\infty)) \in \partial^{2} T=$ $\left\{\left(x_{1}, x_{2}\right) \in \partial T \times \partial T \mid x_{1} \neq x_{2}\right\}$, which, via the action of $F_{k}$, is naturally identified with $\partial^{2} F_{k}$. Conversely, a point $\left(x_{1}, x_{2}\right) \in \partial^{2} F_{k}$ determines two distinct points $x_{1}^{\prime}, x_{2}^{\prime} \in \partial T$. Between these two points, there is a unique (up to orientation preserving reparametrization) oriented geodesic $\ell: \mathbb{R} \rightarrow T$ such that $\ell(\infty)=x_{1}^{\prime}$ and $\ell(-\infty)=x_{2}^{\prime}$. We will use this identification without further remark.

There is fixed point free involution on $\partial^{2} F_{k}$ defined by $\sigma:\left(x_{1}, x_{2}\right) \rightarrow$ $\left(x_{2}, x_{1}\right)$, corresponding to reversing a geodesic's orientation in $T$.

A lamination is a closed $F_{k}$-invariant and $\sigma$-invariant subset $\Lambda \subseteq$ $\partial^{2} F_{k}$. The set of algebraic laminations inherits a Hausdorff topology from $\partial^{2} F_{k}$, which is described in [16]. A nontrivial element $a \in F_{k}$ determines a minimal rational lamination:

$$
\Lambda(a)=\left\{\left(g a^{-\infty}, g a^{+\infty}\right) \cup\left(g a^{+\infty}, g a^{-\infty}\right) \mid g \in F_{k}\right\}
$$

Note that the set $\Lambda(a)$ depends only on the conjugacy class of $a$. Although we will not need them here, we mention that the set of rational laminations consists of finite unions of minimal rational laminations. The most important example of a lamination in what follows is the stable lamination $\Lambda_{+}(\phi)$ associated to a fully irreducible element 
$\phi \in$ Out $F_{k}$, as defined in [7]. ${ }^{1}$ The unstable lamination $\Lambda_{-}(\phi)$ associated to $\phi$ is the stable lamination of $\phi^{-1}$, so that $\Lambda_{-}(\phi)=\Lambda_{+}\left(\phi^{-1}\right)$.

Let $g: G \rightarrow G$ be a train-track representative of $\phi$ with expansion factor $\lambda$. After passing to a power of $g$ if necessary, we can assume that $g$ has a fixed point $x$ contained in the interior of an edge. For some small $\epsilon$-neighborhood $U$ of $x$, we have that $g(U) \supset U$. Fix an isometry $\ell:(-\epsilon, \epsilon) \rightarrow U$ and extend this to the unique isometric immersion $\ell: \mathbb{R} \rightarrow G$ such that $\ell\left(\lambda^{n} t\right)=g^{n}(t)$. This immersion lifts to a collection of geodesics $\tilde{\ell}: \mathbb{R} \rightarrow \widetilde{G}$. Using the identification mentioned above between $\partial^{2} F_{k}$ and the space of geodesics in $\widetilde{G}$, the collection of all geodesics (called leaves) constructed as above determines a closed $F_{k}$-invariant subset of $\partial^{2} F_{k}$ called the stable lamination. It is proved in [7] that this set is well-defined independent of $g$. The leaves of $\Lambda_{+}(\phi)$ are quasi-periodic [7], so that for every $L>0$ there is an $L^{\prime}>L$ such that for every interval $I$ of length $L$ and every interval $I^{\prime}$ of length $L^{\prime}$ there is an element $x \in F_{k}$ such that $x \ell(I) \subseteq \ell\left(I^{\prime}\right)$.

Given a basis $\mathcal{A}$ of $F_{k}$ and a tree $T \in \overline{c v}_{k}$, define the set $L_{\mathcal{A}}^{1}(T)$ as the set of right infinite reduced words $x=x_{1} x_{2} x_{3} \cdots$ in the basis $\mathcal{A}$ such that for some $p \in T$, the sequence of points $\left(x_{1} x_{2} \cdots x_{i}\right) p$ is bounded. The identification of right infinite reduced words in $\mathcal{A}$ with $\partial F_{k}$ identifies $L_{\mathcal{A}}^{1}(T)$ with a subset $L^{1}(T) \subseteq \partial F_{k}$ that is well-defined independent of the choice of basis. Bounded backtracking ensures the existence of a well-defined injective map $\mathcal{Q}: \partial F_{k}-L^{1}(T) \rightarrow \partial T$. Using the injectivity of $\mathcal{Q}$ on $\partial F_{k}-L^{1}(T)$, we associate to any oriented bi-infinite geodesic $\alpha=\left(x_{1}, x_{2}\right) \in \partial^{2} F_{k}-\left(L^{1}(T)\right)^{2}$ an oriented bi-infinite geodesic $\alpha_{T} \subset T$, namely $\alpha_{T}=\left(\mathcal{Q}\left(x_{1}\right), \mathcal{Q}\left(x_{2}\right)\right) \in \partial^{2} T$, if neither endpoint of $\alpha$ is in $L^{1}(T)$; otherwise we define $\alpha_{T}$ to be the empty set. In the latter case, following [33], we say that the geodesic $\alpha$ is $T$-bounded.

In certain cases, the map $\mathcal{Q}: \partial F_{k}-L^{1}(T) \rightarrow \partial T$ extends to a map on $\partial F_{k}$.

Proposition 2.9 ([33], Proposition 3.1). Suppose $T \in \overline{c v}_{k}$ has dense orbits and trivial arc stabilizers (e.g., the stable tree for a fully irreducible outer automorphism). There exists a map $\mathcal{Q}: L^{1}(T) \rightarrow \bar{T}$ to the metric closure $\bar{T}$ of $T$ such that, for any $f: T_{0} \rightarrow T$, where $T_{0} \in c v_{k}$, and any ray $\rho$ in $T_{0}$ representing $x \in L^{1}(T)$, the point $\mathcal{Q}(x)$ belongs to the closure of $f(\rho)$ in $\bar{T}$.

\footnotetext{
${ }^{1}$ Note that in [28], the stable lamination is called the "expanding lamination" and denoted by $\Lambda_{-}$as it is more naturally associated to $T_{-}(\phi)$. See Proposition 2.10 .
} 
Combining this with the previous discussion, we have a map $\mathcal{Q}: \partial F_{k} \rightarrow$ $\bar{T} \cup \partial T$ whenever $T$ has dense orbits and trivial arc stabilizers.

The relation between stable trees and laminations is illustrated by the following.

Proposition 2.10 ([7], Lemma 3.5 (3) \& [33], Corollary 2.3). Suppose that $\phi \in$ Out $F_{k}$ is fully irreducible with stable tree $T_{+}$and unstable lamination $\Lambda_{-}$. Let $\mathcal{Q}: \partial F_{k} \rightarrow \bar{T} \cup \partial T$ be the map defined following Proposition 2.9. Then for any leaf $\ell \in \Lambda_{-}$, we have $\mathcal{Q}(\ell(\infty))=\mathcal{Q}(\ell(-\infty))$.

In light of the above propositions, we can define for any tree $T \in \overline{c v}_{k}$ with dense orbits and trivial arc stabilizers [17]:

$$
L_{\mathcal{Q}}^{2}(T)=\left\{\left(x_{1}, x_{2}\right) \in \partial^{2} F_{k} \mid \mathcal{Q}\left(x_{1}\right)=\mathcal{Q}\left(x_{2}\right)\right\}
$$

where $\mathcal{Q}: \partial F_{k} \rightarrow \bar{T} \cup \partial T$ is the map from Proposition 2.9. With this definition, Proposition 2.10 states that $\Lambda_{-}(\phi) \subseteq L_{\mathcal{Q}}^{2}\left(T_{+}^{\phi}\right)$ where $\phi \in$ Out $F_{k}$ is fully irreducible. If $\phi$ is hyperbolic (i.e., $\phi$ does not have nontrivial periodic conjugacy class) then $L_{\mathcal{Q}}^{2}\left(T_{+}^{\phi}\right)$ is the "diagonal closure" of $\Lambda_{-}(\phi)[30]$.

Missing from the above is a discussion of measured geodesic currents and Dehn twist automorphisms needed for Section 6. We defer their discussion until needed.

\section{Geometric Relative twisting}

Our first definition of relative twisting for Out $F_{k}$ follows closely the original geometric notion for the mapping class group, upon replacing a surface with a suitable 2-complex. This complex, the Guirardel Core for two $F_{k}$-trees $T, T^{\prime}$, is a certain $F_{k}$-invariant subspace $\mathcal{C} \subset T \times T^{\prime}$ (with the diagonal action). We will not need the precise definition of the complex for our purposes; rather, we record in Proposition 3.1 just those properties of $\mathcal{C}$ we do require, together with references.

In the following, if $p$ is a point in $T$, then $\mathcal{C}_{p}=\left\{x^{\prime} \in T^{\prime} \mid\left(p, x^{\prime}\right) \in \mathcal{C}\right\}$; similarly, for $p^{\prime} \in T^{\prime}$, we have $\mathcal{C}_{p^{\prime}}=\left\{x \in T \mid\left(x, p^{\prime}\right) \in \mathcal{C}\right\}$. These sets are called the slices of the core.

Proposition 3.1. Suppose $T, T^{\prime} \in C V_{k}$.

(1) The core $\mathcal{C} \subset T \times T^{\prime}$ is nonempty, connected, closed, $\mathrm{CAT}(0)$, $F_{k}$-invariant and has convex fibers, i.e., the slices $\mathcal{C}_{p}$ and $\mathcal{C}_{p^{\prime}}$ are each convex for all $p \in T$ and $p^{\prime} \in T^{\prime}$. Moreover, $\mathcal{C}$ is the minimal (with respect to inclusion) subset of $T \times T^{\prime}$ with these properties [24, Main Theorem]. 
(2) The quotient $\mathcal{C} / F_{k}$ has finite volume [24, Theorem 8.1]. This volume is called the intersection number, denoted $i\left(T, T^{\prime}\right)$.

(3) For any $p^{\prime} \in T^{\prime}$ that is not a vertex, any arc $\gamma \subset \mathcal{C}_{p^{\prime}}$ is contained in a vanishing path of any change of marking map $f: T \rightarrow T^{\prime}$ [4, Lemma $3.7 \&$ Remark 5.3].

For the complete definition of the core, along with examples, see [4, 24].

Before going further we briefly recall relative twisting for curves on a surface. Let $S$ be a surface of genus at least two, equipped with a hyperbolic metric. We can consider $\pi_{1}(S)$ as a discrete group of isometries of $\mathbb{H}^{2}$, so that $S=\mathbb{H}^{2} / \pi_{1}(S)$. Fix three simple closed curves, $\alpha, \beta, \gamma$, so that $\beta$ and $\gamma$ both intersect $\alpha$. Each of these curves corresponds to a conjugacy class of an element in $\pi_{1}(S)$, and we can assume that all three are geodesics on $S$. Let $S_{\alpha}$ be an annular cover of $S$ corresponding to $\alpha$; that is, the quotient of $\mathbb{H}^{2}$ by the cyclic group generated by a representative of $\alpha$ in $\pi_{1}(S)$. We let $\alpha_{c}$ denote the unique lift of $\alpha$ to $S_{\alpha}$ that is closed. The twist of $\beta$ and $\gamma$ relative to $\alpha$ is defined as the maximum geometric intersection number between $\beta^{\prime}$ and $\gamma^{\prime}$ that intersect $\alpha_{c} \subset S_{\alpha}$, where $\beta^{\prime}$ and $\gamma^{\prime}$ range over lifts of $\beta$ and $\gamma$ to $S_{\alpha}$.

We can reformulate this in terms of the universal cover $\widetilde{S}$, defining the relative twist as follows. Fixing a lift $\tilde{\alpha}$ of $\alpha$ to $\tilde{S}$, the twist of $\beta$ and $\gamma$ relative to $\alpha$ is the maximum number of $\alpha$-translates of $\tilde{\gamma}$ that intersect $\tilde{\beta}$, over all choices of lifts $\tilde{\beta}, \tilde{\gamma}$ of $\beta, \gamma$ that intersect $\tilde{\alpha}$. See Figure 1. This interpretation can be extended to trees in Outer space, using the Guirardel core of $F_{k}$-trees $T$ and $T^{\prime}$ in place of $\tilde{S}$.

The role of the simple closed curves $\beta, \gamma$ is filled by tracks on $\mathcal{C}$, and of the simple closed curve $\alpha$ by the axis of an element of $F_{k}$ in $\mathcal{C}$. A track for $T$ in $\mathcal{C}$ is the set $\{p\} \times \mathcal{C}_{p}$ where $p$ is the midpoint of some edge of $T$; a track for $T^{\prime}$ is defined similarly. We will also use track to refer to the image of a track in the quotient $\mathcal{C} / F_{k}$. We record some elementary properties of tracks.

(1) Every track separates $\mathcal{C}$.

(2) Every track is a finite subtree.

(3) Every track is a convex subset of $\mathcal{C}$.

$\operatorname{As} \mathcal{C}$ is $\operatorname{CAT}(0)$, and every nontrivial element acts hyperbolically, the minset of a nontrivial element $g \in F_{k}$ is isometric to a product $Y \times \mathbb{R}$, where $Y$ is a convex subset of $\mathcal{C}$ [10]. An axis of a nontrivial element $a \in F_{k}$ is a subset of the minset of $a$ of the form $\left\{y_{0}\right\} \times \mathbb{R}$. The element $a$ acts by translation on any of its axes.

Lemmas 3.2 and 3.3 describe the extent to which the intersection number between tracks and axes is well-defined. 


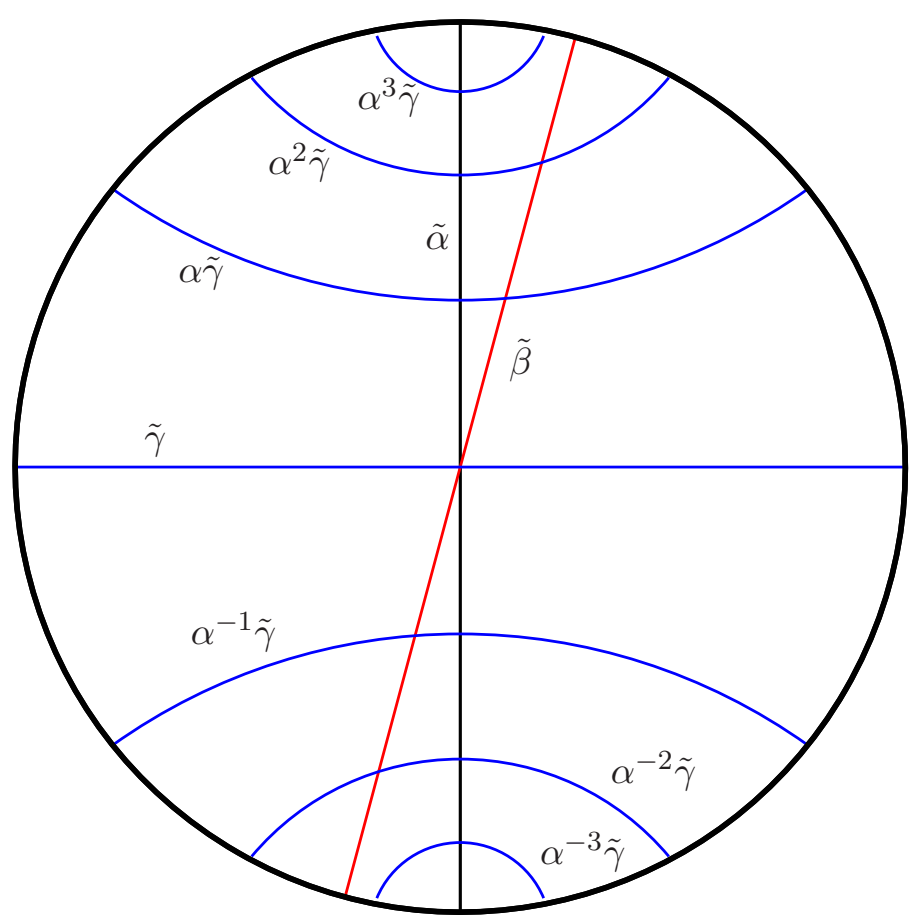

FiguRE 1. The relative twist of $\beta$ and $\gamma$ with respect to $\alpha$ is 5 .

Lemma 3.2. Let $a \in F_{k}$ be a nontrivial element, $T, T^{\prime} \in C V_{k}$ and consider the core $\mathcal{C} \subset T \times T^{\prime}$. If a track $\tau$ in $\mathcal{C}$ intersects an axis of a in $\mathcal{C}$, then it intersects every axis of a in $\mathcal{C}$.

Proof. Let $\tau=\{p\} \times \mathcal{C}_{p}$ be a track for $T$ that intersects an axis for $a$. Let $Y \times \mathbb{R} \subset \mathcal{C}$ be the minset for $a$. As tracks and axes are convex, their intersection is convex as well, and hence connected. Moreover, as tracks are finite, there are $s, t \in \mathbb{R}$ such that $(Y \times \mathbb{R}) \cap \tau \subset Y \times[s, t]$.

Let $\left\{y_{0}\right\} \times \mathbb{R} \subset Y \times \mathbb{R}$ be an axis of $a$ that intersects $\tau$. As $\left(\left\{y_{0}\right\} \times \mathbb{R}\right) \cap$ $\tau$ is connected, we have that $\left\{y_{0}\right\} \times(-\infty, s)$ and $\left\{y_{0}\right\} \times(t, \infty)$ project to different components of $T-\{p\}$. Hence $\tau$ separates $\left\{y_{0}\right\} \times\{s-1\}$ from $\left\{y_{0}\right\} \times\{t+1\}$ in $\mathcal{C}$. If there were an axis, say $\left\{y_{1}\right\} \times \mathbb{R}$, that did not intersect $\tau$, then the concatenation of the fiber-wise paths

$$
\left\{y_{0}\right\} \times\{s-1\} \rightarrow\left\{y_{1}\right\} \times\{s-1\} \rightarrow\left\{y_{1}\right\} \times\{t+1\} \rightarrow\left\{y_{0}\right\} \times\{t+1\}
$$

would be a path that connected $\left\{y_{0}\right\} \times\{s-1\}$ to $\left\{y_{0}\right\} \times\{t+1\}$ avoiding $\tau$, which is a contradiction as $\tau$ separates these points. Thus every axis for $a$ intersects $\tau$.

Lemma 3.3. Let $a \in F_{k}$ be a nontrivial element, $T, T^{\prime} \in C V_{k}$ and consider the core $\mathcal{C} \subset T \times T^{\prime}$. Fix a track $\tau^{\prime}$ for $T^{\prime}$ that intersects an 
axis of a in $\mathcal{C}$. Let $\tau_{0}$ and $\tau_{1}$ be two tracks for $T$ that intersect an axis of a. Then:

$$
|| \tau^{\prime} \cap\langle a\rangle \tau_{0}|-| \tau^{\prime} \cap\langle a\rangle \tau_{1}|| \leq 1
$$

Proof. Suppose $\left|\tau^{\prime} \cap\langle a\rangle \tau_{0}\right|=n$. We will show that $\left|\tau^{\prime} \cap\langle a\rangle \tau_{1}\right| \geq n-1$. The statement of the lemma follows after interchanging $\tau_{0}$ and $\tau_{1}$ and applying the same argument.

Since a track for $T$ and a track for $T^{\prime}$ can intersect at most once in $\mathcal{C}$, the track $\tau^{\prime}$ intersects exactly $n\langle a\rangle$-translates of $\tau_{0}$. We claim that there is an $i$ such that $\tau^{\prime}$ intersects $a^{i+j} \tau_{0}$ for $j=0, \ldots, n-1$. Indeed, this follows as $\tau^{\prime}$ is connected, and as $a^{m} \tau_{0}$ separates $a^{m-r} \tau_{0}$ from $a^{m+s} \tau_{0}$ for all $m$ and positive $r$ and $s$. Replacing $\tau_{0}$ by $a^{i} \tau_{0}$, we can assume that $\tau^{\prime}$ intersects $\tau_{0}, \ldots a^{n-1} \tau_{0}$.

If $\tau_{1}=a^{i} \tau_{0}$ for some $i$, then the statement is obvious. Otherwise, as $\left|\tau^{\prime} \cap\langle a\rangle \tau_{1}\right|$ only depends on the orbit of $\tau_{1}$ under $a$, we can replace $\tau_{1}$ by $a^{i} \tau_{1}$ for some $i$ to assume that $\tau_{1}$ separates $\tau_{0}$ from $a \tau_{0}$.

We claim that $\tau^{\prime}$ intersects $\tau_{1}, \ldots, a^{n-2} \tau_{1}$. Indeed, since $\tau^{\prime}$ is connected and since $a^{i} \tau_{1}$ separates $a^{i} \tau_{0}$ from $a^{i+1} \tau_{0}$, both of which intersect $\tau^{\prime}$ for $i=0, \ldots n-2$, the track $\tau^{\prime}$ must intersect $a^{i} \tau_{1}$.

As $\left|\tau^{\prime} \cap\langle a\rangle \tau\right|=\left|\langle a\rangle \tau^{\prime} \cap \tau\right|$, Lemma 3.3 shows that if $\tau_{0}^{\prime}$ and $\tau_{1}^{\prime}$ are tracks for $T^{\prime}$ that intersect an axis of $a$, and likewise $\tau_{0}$ and $\tau_{1}$ are tracks for $T$ that intersect an axis of $a$, then:

$$
|| \tau_{0}^{\prime} \cap\langle a\rangle \tau_{0}|-| \tau_{1}^{\prime} \cap\langle a\rangle \tau_{1}|| \leq 2
$$

With this bound we can define the relative twist number. By $\operatorname{Tr}_{a}(T)$ we denote the set of tracks for $T$ in $\mathcal{C}$ that intersect an (and hence every) axis of $a$. We define the set $\operatorname{Tr}_{a}\left(T^{\prime}\right)$ similarly.

Definition 3.4. Given $T, T^{\prime} \in C V_{k}$ and a nontrivial element $a \in F_{k}$, define the twist of $T$ and $T^{\prime}$ relative to a as:

$$
\tau_{a}\left(T, T^{\prime}\right)=\max _{\tau^{\prime} \in \operatorname{Tr}_{a}\left(T^{\prime}\right), \tau \in T r_{a}(T)}\left|\tau^{\prime} \cap\langle a\rangle \tau\right| .
$$

We remark that this number is always finite. Indeed, as tracks are finite, the quantities we are maximizing over are finite. Then by the above discussion, there are only finitely many distinct possibilities for these numbers.

The significance of the relative twist number to the geodesic in $C V_{k}$ connecting two marked graphs is the following, to be used in Section 5 to prove Theorem 5.2:

Proposition 3.5. Suppose $G, G^{\prime} \in C V_{k}$ with $d=d_{L}\left(G, G^{\prime}\right)$ such that $\tau_{a}\left(G, G^{\prime}\right) \geq n$ for some nontrivial $a \in F_{k}$. Then for every change of 
marking map $g: G \rightarrow G^{\prime}$, there is a vanishing path $\gamma \subset G$ that $n$-covers $a$.

Proof. Let $\widetilde{G}$ and $\widetilde{G}^{\prime}$ be the universal covers of $G$ and $G^{\prime}$ respectively, and consider the core $\mathcal{C} \subset \widetilde{G} \times \widetilde{G}^{\prime}$. Fix an axis of $a$, and tracks $\tau \in$ $\operatorname{Tr}_{a}(\widetilde{G}), \tau^{\prime} \in \operatorname{Tr}_{a}\left(\widetilde{G}^{\prime}\right)$ such that $\tau_{a}\left(G, G^{\prime}\right)=\left|\tau^{\prime} \cap\langle a\rangle \tau\right|$.

Let $m_{0}$ and $m_{1}$ be the least and greatest integer, respectively, such that $\tau^{\prime} \cap a^{m_{0}} \tau \neq \emptyset$ and $\tau^{\prime} \cap a^{m_{1}} \tau \neq \emptyset$. Thus $\tau_{a}\left(G, G^{\prime}\right)=m_{1}-m_{0} \geq n$. Denote $x_{0}=\tau^{\prime} \cap a^{m_{0}} \tau$ and $x_{1}=\tau^{\prime} \cap a^{m_{1}} \tau$ and let $\rho$ be the path in $\tau^{\prime}$ connecting $x_{0}$ to $x_{1}$. As $\tau^{\prime}=\mathcal{C}_{p^{\prime}} \times\left\{p^{\prime}\right\}$ for some point $p^{\prime} \in \widetilde{G}^{\prime}$, we can consider $\rho$ as a path in $\mathcal{C}_{p^{\prime}} \subset \widetilde{G}$. Notice that the endpoints of $\rho$, also denoted $x_{0}$ and $x_{1}$, are on the axis for $a$, and that $a^{m_{1}-m_{0}} x_{0}=x_{1}$. Thus $a$ is $n$-covered by $\rho$. By Proposition 3.1(3), the path $\rho$ is contained in a vanishing path $\gamma$ for any change of marking map $G \rightarrow G^{\prime}$. As $\gamma$ contains $\rho$, the vanishing path $\gamma n$-covers $a$ as well.

\section{Algebraic Relative twisting}

In this section we give our algebraic interpretation of relative twisting and develop some consequences that will be useful for applications in later sections. The key result is Proposition 4.8, which is used to prove Theorem 5.3.

Definition 4.1. Given $T \in \overline{c v}_{k}$, a lamination $\Lambda \subset \partial^{2} F_{k}$, and an element $a \in F_{k}$, if $\ell_{T}(a) \neq 0$, then we define the twist of $T$ and $\Lambda$ relative to $a$ to be:

$$
\tau_{a}(T, \Lambda)=\sup \left\{\frac{L_{T}\left(\alpha_{T} \cap T^{\langle a\rangle}\right)}{\ell_{T}(a)} \mid \alpha \in \Lambda\right\} .
$$

If $\ell_{T}(a)=0$, then define $\tau_{a}(T, \Lambda)=0$.

Recall that given $\alpha=\left(x_{1}, x_{2}\right) \in \partial^{2} F_{k}$, we have that $\alpha_{T}$ is equal to $\left(\mathcal{Q}\left(x_{1}\right), \mathcal{Q}\left(x_{2}\right)\right) \in \partial^{2} T$ if $\alpha$ is not $T$-bounded, and is equal to the empty set otherwise. We insist that $L_{T}(\emptyset)=0$.

Remark 4.2. We allow for the possibility that $\tau_{a}(T, \Lambda)=\infty$. This occurs in particular for the rational lamination $\Lambda(a)$, when $\ell_{T}(a) \neq 0$.

Central to our analysis is the following proposition:

Proposition 4.3. Suppose $a \in F_{k}, T \in \overline{c v}_{k}$, and that $\Lambda$ is a lamination containing no $T$-bounded geodesic. If $\left\{T_{i}\right\}$ is a sequence of trees in $c v_{k}$ converging to $T$, and $\left\{\Lambda_{i}\right\}$ is a sequence of laminations converging to $\Lambda$, then:

$$
\lim _{i \rightarrow \infty} \tau_{a}\left(T_{i}, \Lambda_{i}\right) \geq \tau_{a}(T, \Lambda)
$$


Proof. The proposition is obviously true when $\ell_{T}(a)=0$, and so we assume that $\ell_{T}(a)>0$.

We proceed with the following:

Claim. If $T \in \overline{c v}_{k}$, and if $\alpha, \beta \in \partial^{2} F_{k}$ are not $T$-bounded and $\alpha(\infty), \alpha(-\infty), \beta(\infty), \beta(-\infty)$ are four distinct points, then for sufficiently close $T^{\prime} \in c v_{k}$, we have $L_{T^{\prime}}\left(\alpha_{T^{\prime}} \cap \beta_{T^{\prime}}\right)$ close to $L_{T}\left(\alpha_{T} \cap \beta_{T}\right)$.

Proof. As $\mathcal{Q}$ is injective on $\partial F_{k}-L^{1}(T)$, we have that $\alpha_{T} \cap \beta_{T}$ is compact set.

Fix a tree $T_{0} \in c v_{k}$, a map $f: T_{0} \rightarrow T$ that is linear on edges, and elements $a_{i}^{ \pm} \in T_{0}$ so that $\left[a_{i}^{-}, a_{i}^{+}\right] \rightarrow \alpha_{T_{0}}$. Then $\alpha_{i}=\left[f\left(a_{i}^{-}\right), f\left(a_{i}^{+}\right)\right] \rightarrow$ $\alpha_{T}$; in particular, the overlap of $\alpha_{i}$ and $\alpha_{T}$ can be made arbitrarily large. Similarly define $b_{i}^{ \pm} \in T_{0}$ and $\beta_{i}=\left[f\left(b_{i}^{-}\right), f\left(b_{i}^{+}\right)\right]$so that, as before, we have $\beta_{i} \rightarrow \beta_{T}$.

Fix a $T^{\prime} \in c v_{k}$ and an equivariant map $f^{\prime}: T_{0} \rightarrow T^{\prime}$, linear on edges. As before, we have $\alpha_{i}^{\prime}=\left[f^{\prime}\left(a_{i}^{-}\right), f^{\prime}\left(a_{i}^{+}\right)\right] \rightarrow \alpha_{T^{\prime}}$ and $\beta_{i}^{\prime}=$ $\left[f^{\prime}\left(b_{i}^{-}\right), f^{\prime}\left(b_{i}^{+}\right)\right] \rightarrow \beta_{T^{\prime}}$.

Now choose $n$ large enough so that each of $\alpha_{n} \cap \alpha_{T}$ and $\beta_{n} \cap \beta_{T}$ contains $\alpha_{T} \cap \beta_{T}$. Then increase $n$ if necessary so that $\alpha_{n}^{\prime} \cap \alpha_{T^{\prime}}$ and $\beta_{n}^{\prime} \cap \beta_{T^{\prime}}$ each contain $\alpha_{T^{\prime}} \cap \beta_{T^{\prime}}$. For sufficiently close trees $T, T^{\prime}$, the lengths $L_{T}\left(\alpha_{n} \cap \beta_{n}\right)$ and $L_{T^{\prime}}\left(\alpha_{n}^{\prime} \cap \beta_{n}^{\prime}\right)$ of the overlaps are close $[11,25]$. By choice of $n$, we have $L_{T}\left(\alpha_{n} \cap \beta_{n}\right)=L_{T}\left(\alpha_{T} \cap \beta_{T}\right)$ and $L_{T^{\prime}}\left(\alpha_{n}^{\prime} \cap \beta_{n}^{\prime}\right)=$ $L_{T^{\prime}}\left(\alpha_{T^{\prime}} \cap \beta_{T^{\prime}}\right)$. The claim follows.

We are now prepared to complete the proof of the proposition. First assume that $\tau_{a}(T, \Lambda) \neq \infty$. This implies that no geodesic in $\Lambda$ has $a^{+\infty}$ or $a^{-\infty}$ as an endpoint and so we can use the above claim. Let $\epsilon$ be small and choose a geodesic $\alpha \in \Lambda$ so that:

$$
L_{T}\left(\alpha_{T} \cap T^{\langle a\rangle}\right) / \ell_{T}(a)>\tau_{a}(T, \Lambda)-\epsilon .
$$

Then by the above:

$$
\frac{L_{T^{\prime}}\left(\alpha_{T^{\prime}} \cap T^{\prime\langle a\rangle}\right)}{\ell_{T^{\prime}}(a)}>\frac{L_{T}\left(\alpha_{T} \cap T^{\langle a\rangle}\right)}{\ell_{T}(a)}-\epsilon>\tau_{a}(T, \Lambda)-2 \epsilon
$$

for $T^{\prime}$ sufficiently close to $T$. For $\Lambda^{\prime}$ sufficiently close to $\Lambda$, there exists $\alpha^{\prime} \in \Lambda^{\prime}$ so that $\alpha_{T^{\prime}} \cap T^{\prime\langle a\rangle} \subset \alpha_{T^{\prime}}^{\prime}$. Thus:

$$
\tau_{a}\left(T^{\prime}, \Lambda^{\prime}\right) \geq \frac{L_{T^{\prime}}\left(\alpha_{T^{\prime}} \cap T^{\prime\langle a\rangle}\right)}{\ell_{T^{\prime}}(a)}>\tau_{a}(T, \Lambda)-2 \epsilon
$$

for $T^{\prime}$ sufficiently close to $T$ and $\Lambda^{\prime}$ sufficiently close to $\Lambda$. Since we obtain such an inequality for every $\epsilon$, the proposition holds. 
Suppose $\tau_{a}(T, \Lambda)=\infty$. If $a^{+\infty}$ or $a^{-\infty}$ is an endpoint of a geodesic in $\Lambda$, then $\tau_{a}\left(T^{\prime}, \Lambda\right)=\infty$ for all trees $T^{\prime} \in c v_{k}$. Else, we have that for, for every $M>0$ there is a geodesic $\alpha \in \Lambda$ so that:

$$
\infty>L_{T}\left(\alpha_{T} \cap T^{\langle a\rangle}\right) / \ell_{T}(a)>M .
$$

Then arguing in a similar fashion as above, we have for $T^{\prime}$ sufficiently close to $T$ and $\Lambda^{\prime}$ sufficiently close to $\Lambda$ :

$$
\tau_{a}\left(T^{\prime}, \Lambda^{\prime}\right) \geq \frac{L_{T^{\prime}}\left(\alpha_{T^{\prime}} \cap T^{\prime\langle a\rangle}\right)}{\ell_{T^{\prime}}(a)}>\frac{M}{2} .
$$

Since we obtain such an inequality for every $M$, the proposition holds.

Remark 4.4. Examples of tree, lamination pairs satisfying the hypotheses of Proposition 4.3 are:

(1) $T_{+}(\phi), \Lambda_{+}(\phi)$ where $\phi \in$ Out $F_{k}$ is fully irreducible,

(2) $T, \Lambda$ where $T \in c v_{k}$ and $\Lambda$ is any lamination, and

(3) $T, \Lambda(a)$ whenever $\ell_{T}(a) \neq 0$.

For a fully irreducible element $\phi$ with large twist $\tau_{a}\left(T_{-}(\phi), \Lambda_{-}(\phi)\right)$ for some nontrivial $a \in F_{k}$, our goal is to locate a train-track $G_{0}$ of $\phi$ with a vanishing path that $n$-covers $a$, similar to Proposition 3.5. Our tool to produce such a path is Proposition 2.8. First we see how to use the lamination to get the required setup.

Lemma 4.5. Suppose $\phi \in$ Out $F_{k}$ is fully irreducible, $g: G \rightarrow G$ is a train-track representative for $\phi, T_{+} \in \overline{c v}_{k}$ is the stable tree for $\phi$, $f_{g}: \widetilde{G} \rightarrow T_{+}$is the induced map from Theorem 2.4, and $\ell: \mathbb{R} \rightarrow \widetilde{G}$ is a leaf of the unstable lamination $\Lambda_{-}$. Then for all $I \subset \mathbb{R}$, there exists $I^{\prime}=[x, y] \subset \mathbb{R}$ such that $I \subseteq I^{\prime}$ and $f_{g}(\ell(x))=f_{g}(\ell(y))$.

Proof. We claim that for any $L \geq 0$, there is an interval $[a, b] \subseteq \mathbb{R}$ such that $|b-a| \geq L$ and $f_{g}(\ell(a))=f_{g}(\ell(b))$. The lemma follows: by the quasi-periodicity of $\ell$, there is then an interval $I_{0}=[a, b] \subset \mathbb{R}$ such that $f_{g}(\ell(a))=f_{g}(\ell(b))$ and $x \ell(I) \subseteq \ell\left(I_{0}\right)$. Setting $I^{\prime}=\ell^{-1}\left(x^{-1} \ell\left(I_{0}\right)\right)$ completes the proof. We must then just establish the claim.

Since $\ell$ is a leaf of the unstable lamination, we have $\mathcal{Q}(\ell(-\infty))=$ $\mathcal{Q}(\ell(\infty))$. There are sequences $a_{i} \rightarrow-\infty$ and $b_{i} \rightarrow \infty$ such that $f_{g}\left(\ell\left(a_{i}\right)\right) \rightarrow \mathcal{Q}(\ell(-\infty))=\mathcal{Q}(\ell(\infty))$ and $f_{g}\left(\ell\left(b_{i}\right)\right) \rightarrow \mathcal{Q}(\ell(\infty))[33$, Lemma 3.4]. Now we have two cases, either the sequences $\left\{f_{g}\left(\ell\left(a_{i}\right)\right)\right\}$ and $\left\{f_{g}\left(\ell\left(b_{i}\right)\right)\right\}$ are in the same component of $\bar{T}_{+}-\{\mathcal{Q}(\infty)\}$ or they are not.

If the sequences are in the same component, choose $n$ with $\left|b_{n}-a_{n}\right|>$ $L$. The arc $\alpha$ connecting $f_{g}\left(a_{n}\right)$ to $f_{g}\left(b_{n}\right)$ is then disjoint from $\mathcal{Q}(\ell(\infty))$, 
and there is a unique point $p \in \alpha$ which is closest to $\mathcal{Q}(\ell(\infty))$. As $\bar{T}_{+}$is an $\mathbb{R}$-tree, $p$ is on the geodesic $\left[f_{g}\left(a_{n}\right), \mathcal{Q}(\ell(-\infty))\right]$. Then by continuity of $f \ell$, there is an $a^{\prime} \leq a_{n}$ such that $f_{g}\left(a^{\prime}\right)=p$. Likewise, there is a $b^{\prime} \geq b_{n}$ such that $f_{g}\left(b^{\prime}\right)=p$. Thus the inteveral $\left[a^{\prime}, b^{\prime}\right]$ satisfies the claim.

Now suppose the sequences are not in the same component. If $f_{g}(\ell(\mathbb{R}))$ crosses the point $\mathcal{Q}(\ell(\infty))$ infinitely many times, then we can find a sequence of points $a_{i}, b_{i} \in \mathbb{R}$ such that $f_{g}\left(\ell\left(a_{i}\right)\right)=f_{g}\left(\ell\left(b_{i}\right)\right)=$ $\mathcal{Q}(\ell(\infty))$ such that $\left|b_{i}-a_{i}\right| \rightarrow \infty$. Indeed, there is a lower bound on the distance between two pre-images of $\mathcal{Q}(\infty)$ in $\mathbb{R}$ as every edge of $\widetilde{G}$ is isometrically embedded by $f_{g}$. For large enough $i$, the interval $\left[a_{i}, b_{i}\right]$ satisfies the claim.

Finally, suppose $f_{g}(\ell(\mathbb{R}))$ crosses $\mathcal{Q}(\ell(\mathbb{R}))$ only finitely many times. Let $a$ be the smallest number such that $f_{g}(\ell(a))=\mathcal{Q}(\ell(\infty))$. Then arguing as in the first case using sequences $a_{i} \rightarrow-\infty$ and $b_{i} \rightarrow a$ $\left(b_{i}<a\right)$ we can find the desired inteveral.

In the next proposition, we find a candidate vanishing path in a train-track $G$ that folds over $a$ several times. The technicalities in its proof arise from the fact that, as the hypothesis concerns the unstable lamination, we must first find a large power of $a$ covered by a leaf of the lamination in a train-track map for the inverse $\phi^{-1}$ of $\phi$. Care is then required in mapping this leaf over to a train-track for $\phi$, as there might be excessive cancellation. We resolve this difficulty by applying powers of $\phi^{-1}$, so that the length of $a$ dominates any such cancellation.

Proposition 4.6. Suppose that $\phi$ is fully irreducible with unstable tree $T_{-}$and lamination $\Lambda_{-}$, with $\tau_{a}\left(T_{-}, \Lambda_{-}\right) \geq n+2$ for some $a \in F_{k}$. Then there exists a train-track graph $G \in C V_{k}$ for $\phi$ and a leaf of the unstable lamination $\ell: \mathbb{R} \rightarrow \widetilde{G}$ such that for all $L>0$, there is a finite interval $I \subset \mathbb{R}$ such that $[\ell(I)] \dagger_{L} n$-covers $a$.

Proof. Let $H \in C V_{k}$ be a train-track graph for $\phi^{-1}$ with train track representative $h: H \rightarrow H$ and $G \in C V_{k}$ a train track graph for $\phi$ with train-track map $g: G \rightarrow G$. Fix Lipschitz homotopy equivalences $\kappa: H \rightarrow G$ and $\kappa^{\prime}: G \rightarrow H$ representing the change in markings. Thus the following diagram is commutative up to homotopy:

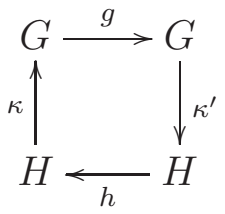


Notice that $\kappa$ lifts to $\widetilde{\kappa}: \widetilde{H} \rightarrow \widetilde{G}$, with bounded backtracking. In particular, we can pick a constant $C$ such that if a path $\gamma \subset H$ has length at least $C$, then $\kappa(\gamma)$ is not homotopically trivial relative to its endpoints.

As $\tau_{a}\left(T_{-}, \Lambda_{-}\right) \geq n+2$, Proposition 4.3 implies that $\tau_{a}\left(\widetilde{H}_{0}, \Lambda_{-}\right) \geq$ $n+2$, where $H_{0}=H \phi^{-M}$ for some large $M$. Define $G_{0}=G \phi^{-M}$. Now there is a leaf $\ell: \mathbb{R} \rightarrow \widetilde{H}_{0}$ of the unstable lamination and an interval $I_{0} \subset \mathbb{R}$ such that $\ell\left(I_{0}\right) \subset \widetilde{H}_{0}^{\langle a\rangle}$ and $L_{\widetilde{H}_{0}}\left(\ell\left(I_{0}\right)\right) \geq(n+2) \ell_{\widetilde{H}_{0}}(a)$. Notice that this implies that the loop representing the conjugacy class of $a$ in $H_{0}$ is legal with respect to $h$. Then if we let $\lambda$ be the expansion factor for $\phi^{-1}$, and let $d=\ell_{\widetilde{H}_{0}}(a)$, we have $\ell_{\widetilde{H}_{0} \phi^{-m}}(a)=\lambda^{m} d$. Let $N$ be such that $\lambda^{N} d \geq C$. Define $G_{1}=G_{0} \phi^{-N}$ and $H_{1}=H_{0} \phi^{-N}$.

Fix $L>0$ and let $L^{\prime}$ be such that if we have paths $\gamma \subset \gamma^{\prime}$ in $H_{0}$ and $\gamma$ has length at least $L^{\prime}$ then the path $\kappa(\gamma)$ intersects $\left[\kappa\left(\gamma^{\prime}\right)\right]$ in a path of length at least $L$ (necessarily a subpath of $\left[\kappa\left(\gamma^{\prime}\right)\right]$, but not necessarily a subpath of $[\kappa(\gamma)]$ ). Indeed, such an $L^{\prime}$ exists as $\kappa$ is a quasi-isometry and the graph $G_{0}$ has valence bounded by $2 k$. Finally, extend $I_{0}$ to $I=I_{1} \cup I_{0} \cup I_{2} \subset \mathbb{R}$ by including intervals $I_{1}$ and $I_{2}$ of length at least $\left(L^{\prime}+C\right) / \lambda^{N}$.

Notice that the lengths of $h^{N}\left(\ell\left(I_{1}\right)\right)$ and $h^{N}\left(\ell\left(I_{2}\right)\right)$ in $H_{1}$ are at least $L^{\prime}+C$. Moreover, the initial subsegment $\iota \subset h^{N}\left(\ell\left(I_{1}\right)\right)$ of length $L^{\prime}$ maps by $\kappa$ to a segment in $G_{1}$ that intersects $\left[\kappa h^{N} \ell(I)\right]$ in a path of length at least $L$, so that $\kappa(\iota)$ does not cancel with $\kappa h^{N} \ell\left(I_{0}\right)$, as $\iota$ and $h^{N} \ell\left(I_{0}\right)$ are separated in $h^{N} \ell(I)$ by a subsegment of length at least $C$. Similarly for the terminal subsegment of $h^{N}\left(I_{2}\right)$.

Recall $\ell\left(I_{0}\right)(n+2)$-covers $a$ in $H_{0}$. This clearly implies that $h^{N} \ell\left(I_{0}\right)$ $(n+2)$-covers $a$ in $H_{1}$ as all of the paths are legal with respect to $h$. Thus $h^{N} \ell\left(I_{0}\right)$ contains a subpath whose image in $H_{1}$ represents the conjugacy class of $a^{n+2}$. As $\ell_{\widetilde{H}_{1}}(a) \geq C$, a subsegment of length $n \ell_{\widetilde{G}_{1}}(a)$ in $\left[\kappa h^{N} \ell\left(I_{0}\right)\right]$ survives after tightening $\left[\kappa h^{N} \ell\left(I_{1}\right)\right] \cdot\left[\kappa h^{N} \ell\left(I_{0}\right)\right] \cdot\left[\kappa h^{N} \ell\left(I_{2}\right)\right]$ to get $\left[\kappa h^{N} \ell(I)\right]$. Now tighten $\kappa \ell$ to get a leaf of the unstable lamination $\ell_{1}: \mathbb{R} \rightarrow \widetilde{G}_{1}$. Thus $L_{\widetilde{G}_{1}}\left(\left[\ell_{1}(I)\right] \dagger_{L} \cap \widetilde{G}_{1}^{\langle a\rangle}\right) \geq n \ell_{\widetilde{G}_{1}}(a)$ and hence $\left[\ell_{1}(I)\right] \dagger_{L}$ $n$-covers $a$.

Proposition 4.8 will now follow relatively quickly once we observe the following consequence of Proposition 4.6.

Corollary 4.7. Suppose that $\phi$ is fully irreducible with unstable tree $T_{-}$and lamination $\Lambda_{-}$, such that $\tau_{a}\left(T_{-}, \Lambda_{-}\right) \geq n+2$ for some $a \in F_{k}$. Then there exists a train-track $G \in C V_{k}$ for $\phi$, with train-track map $g: G \rightarrow G$, and a path $\gamma=[x, y] \subset \widetilde{G}$ such that:

(1) $\gamma n$-covers $a$; and 
(2) $f_{g}(x)=f_{g}(y)$ where $f_{g}: \widetilde{G} \rightarrow T_{+}$is the induced map (see Theorem 2.4) from $\widetilde{G}$ to $T_{+}$, the stable tree for $\phi$.

Proof. Let $G$ be the train-track graph given by Proposition 4.6, and let $f_{g}: \widetilde{G} \rightarrow T_{+}$be the induced map. Let $\ell: \mathbb{R} \rightarrow \widetilde{G}$ be the leaf of the unstable lamination and $I \subset \mathbb{R}$ the interval produced by Proposition 4.6, for $L=B B T\left(f_{g}\right)+1$. For this $I$, let $I^{\prime}$ be the interval given by Lemma 4.5.

We claim that $\gamma=\left[\ell\left(I^{\prime}\right)\right]$ satisfies the conclusion of the corollary. Indeed by Lemma 2.3, as $[\ell(I)] \dagger_{L}$ contains a path in the axis of $a$ of length $n \ell_{\widetilde{G}}(a)$, so does $\gamma=\left[\ell\left(I^{\prime}\right)\right]$. By construction, $f_{g}$ identifies the endpoints of $\gamma$.

Proposition 2.8 applied to the train-track $G$ and the path $\gamma$ of Corollary 4.7 give the following:

Proposition 4.8. Suppose $\phi$ is a fully irreducible element with unstable tree $T_{-}$and lamination $\Lambda_{-}$such that $\tau_{a}\left(T_{-}, \Lambda_{-}\right) \geq n+2$ for some $a \in F_{k}$. Then there exists a train-track $G \in C V_{k}$ for $\phi$, with train-track map $g: G \rightarrow G$, and a vanishing path $\gamma \subset G$ that $n$-covers a.

\section{Finding SMALl CYCLES}

With our definition of relative twist, we can prove the analogue of a special case of Rafi's characterization of short curves along geodesics in Teichmüller space [37].

Proposition 5.1. Suppose $G, G^{\prime} \in C V_{k}, f: G \rightarrow G^{\prime}$ is a change of marking map with minimal slope, $d=d_{L}\left(G, G^{\prime}\right)$ and $a \in F_{k}$. If there is a vanishing path $\gamma \subset G$ that $(n+2)$-covers a, then there is a geodesic $\alpha:[0, d] \rightarrow C V_{k}$ such that $\alpha(0)=G, \alpha(d)=G^{\prime}$ and for some $t \in[0, d]$, we have $\ell_{\alpha(t)}(a) \leq 1 / n$.

Proof. Shrinking the edges of $G$ such that each edge is stretched by exactly $e^{d}$ results in a marked graph $G_{0}$ and provides a geodesic $\alpha:\left[0, d_{0}\right] \rightarrow$ $C V_{k}$ such that $\alpha(0)=G, \alpha\left(d_{0}\right)=G_{0}$ and $d=d_{L}\left(G_{0}, G^{\prime}\right)+d_{0}$. Denote the induced map $G_{0} \rightarrow G^{\prime}$ by $f$.

Consider the graph $H_{a}=\widetilde{G}_{0}^{\langle a\rangle} /\langle a\rangle$ and the map $h_{G^{\prime}}: H_{a} \rightarrow G^{\prime}$ which is the composition of the immersion $H_{a} \rightarrow G_{0}$ with the map $f: G_{0} \rightarrow G^{\prime}$. By appropriately subdividing and folding the graph $H_{a}$, after pruning we obtain a graph immersion $H_{a}^{\prime} \rightarrow G^{\prime}$. Now choose a folding path based at $G_{0}$ whose folds correspond to the folds performed on $H_{a}$. The end of the folding path is a graph $G_{1}$ in which $H_{a}^{\prime}$ is immersed. 
Let $d_{1}=d_{L}\left(G_{0}, G_{1}\right)$. As $f$ has minimal slope, the above path extends $\alpha$ to a geodesic $\alpha:\left[0, d_{1}\right] \rightarrow C V_{k}$ such that $\alpha(0)=G$ and $\alpha\left(d_{1}\right)=G_{1}$ [23, Theorem 5.5]. Further $d_{L}\left(G_{1}, G^{\prime}\right)=d-d_{1}$. Denote the induced $\operatorname{map} G_{1} \rightarrow G^{\prime}$ by $f_{1}$.

The geodesic segment $\alpha$ can further be extended to a geodesic by folding $G_{1}$. The image of $\gamma$ in $G_{1}$ (which we denote by $\gamma_{1}$ ) is a vanishing path for the map $f_{1}$.

Claim. The path $\gamma_{1} \subset G_{1} n$-covers $a$.

Proof of Claim. Consider the graph $\widetilde{G}_{0} /\langle a\rangle$. This graph consists of a collection of trees attached to $H_{a}$. We consider the graph $H_{a}$ as oriented counterclockwise and decompose $H_{a}$ into subsegments $\delta_{1} \epsilon_{1} \cdots \delta_{\ell} \epsilon_{\ell}$ where the $\delta_{i}$ are the maximal subsegments that remain upon folding $H_{a} \rightarrow H_{a}^{\prime}$ and pruning. The images of the $\epsilon_{i}$ are what get pruned. There is a lift of $\gamma$ to $\widetilde{G}_{0} /\langle a\rangle$ that decomposes into subpaths $\gamma=\beta_{0} \alpha \beta_{1}$ where $\beta_{0}$ and $\beta_{1}$ are embedded and $\alpha$ is the immersed path that covers $H_{a} n+2$ times.

When folding the segments $\epsilon_{i}$, the initial part of $\beta_{1}$ may (by equivariance) become identified with some portion of $H_{a}$. We are interested in bounding how much is identified with the terminal portion of $\alpha$ as this could reduce the amount of $\gamma_{1}$ that covers $a$. We will show that the portion of $\alpha$ identified is a segment of $H_{a}$. In other words, we can reduce this amount by at most 1 .

Without loss of generality we assume that $\beta_{1}$ only intersects $H_{a}$ in a single vertex. Suppose this vertex is in $\delta_{i}$ and consider performing the folds in $\epsilon_{i}$. After folding $\epsilon_{i}$, the subsegment of the terminal part of $\alpha$ identified with the initial part of $\beta_{1}$ either heads counterclockwise from $v_{0}$ (which we are not concerned with as this adds to the amount by which $\gamma_{1}$ covers $a$ ) or it is contained in the union of $\delta_{i}$ and $\epsilon_{i}$. Indeed, we can just check locally that when folding two edges $e_{1}$ and $e_{2}$ in $\epsilon_{i}$ together in $H_{a}$ that $\beta_{1}$ cannot fold past (in the clockwise direction) the image of their terminal vertices. This involves a few cases depending on the relative positions of $\beta_{1}, e_{1}$ and $e_{2}$; all of which are easily verified.

Similarly, if $\beta_{1}$ only intersects $H_{a}$ in a vertex of $\epsilon_{i}$, then we find the the subsegment of the terminal portion of $\alpha$ that is identified with $\beta_{1}$ either heads counterclockwise from $v_{0}$ or it is contained in $\epsilon_{i}$.

Thus when performing the folds in $H_{a} \rightarrow H_{a}^{\prime}$, the initial portion of $\beta_{1}$ is identified at most one copy of $H_{a}$. Likewise, the same holds for the terminal portion of $\beta_{0}$. Therefore the image path $\gamma_{1} n$-covers $a$.

As a consequence of the claim, we have $\ell_{G^{\prime}}\left(a^{n}\right) \leq B B T\left(f_{1}\right)$. Since $H_{a}^{\prime}$ is immersed in every graph along the folding path between $G_{1}$ and 
$G^{\prime}$, we have $\ell_{G^{\prime}}\left(a^{n}\right)=\operatorname{Lip}\left(f_{1}\right) \ell_{G_{1}}\left(a^{n}\right)$, so that

$$
\ell_{G_{1}}\left(a^{n}\right)=\ell_{G^{\prime}}\left(a^{n}\right) / \operatorname{Lip}\left(f_{1}\right) \leq B B T\left(f_{1}\right) / \operatorname{Lip}\left(f_{1}\right) \leq 1
$$

and so $\ell_{G_{1}}(a) \leq \frac{1}{n}$.

Combining Proposition 5.1 with Proposition 3.5 we get the first of the main results of this paper.

Theorem 5.2. Suppose $G, G^{\prime} \in C V_{k}$ with $d=d_{L}\left(G, G^{\prime}\right)$ such that $\tau_{a}\left(G, G^{\prime}\right) \geq n+2$ for some $a$. Then there is a geodesic $\alpha:[0, d] \rightarrow C V_{k}$ such that $\alpha(0)=G$ and $\alpha(d)=G^{\prime}$ and for some $t \in[0, d]$, we have $\ell_{\alpha(t)}(a) \leq 1 / n$. In other words, $\alpha([0, d]) \cap C V_{k}^{1 / n}(a) \neq \emptyset$.

Additionally, combining Proposition 5.1 with Proposition 4.8 we get the second of the main results of this paper.

Theorem 5.3. Suppose $\phi \in$ Out $F_{k}$ is fully irreducible, with unstable tree $T_{-}$and lamination $\Lambda_{-}$such that $\tau_{a}\left(T_{-}, \Lambda_{-}\right) \geq n+4$ for some $a \in F_{k}$. Then given any train-track $G$, there is an axis $\mathcal{L}_{\phi}$ for $\phi$ that contains $G$ and a graph $G_{0}$ such that $\ell_{\widetilde{G}_{0}}(a) \leq 1 / n$. In other words, $\mathcal{L}_{\phi} \cap C V_{k}^{1 / n}(a) \neq \emptyset$.

\section{EXAMPLE}

Here we present an application of Theorem 5.3 in which we describe the asymptotic behavior of the translation length in $C V_{k}$ of certain elements of Out $F_{k}$, given as products $\phi_{n}=\delta_{1}^{n} \delta_{2}^{-n}$ of powers of Dehn twists $\delta_{1}, \delta_{2}$. These types of outer automorphisms were considered in [13] and used in [12] to show that there is no homological obstruction to full irreducibility. We briefly recall the setup here; for more details consult either of the references $[12,13]$.

6.1. Dehn twists. A cyclic tree is a Bass-Serre tree associated to a splitting of $F_{k}$ over $\mathbb{Z}$, either as an amalgamated free product or as an HNN-extension. To such a tree is an associated (outer) automorphism called a Dehn twist. Given $F_{k}=A *_{\langle c\rangle} B$ we define the Dehn twist automorphism $\delta_{c}$ of $F_{k}$ by:

$$
\begin{array}{ll}
\forall a \in A & \delta_{c}(a)=a \\
\forall b \in B & \delta_{c}(b)=c b c^{-1} .
\end{array}
$$

Likewise, given $F_{k}=A *_{\mathbb{Z}}=\left\langle A, t \mid t^{-1} c t=c^{\prime}\right\rangle$ for $c, c^{\prime} \in A$, we define the Dehn twist $\delta_{c}$ of $F_{k}$ by:

$$
\begin{aligned}
\forall a \in A & \delta_{c}(a)=a \\
& \delta_{c}(t)=c t .
\end{aligned}
$$


Two cyclic trees $T_{1}$ and $T_{2}$ fill if their associated Dehn twists $\delta_{1}, \delta_{2}$ do not have any common invariant conjugacy classes of proper free factors or cyclic subgroups. As mentioned above, if $T_{1}$ and $T_{2}$ fill, then for large enough $n$, the element $\delta_{1}^{n} \delta_{2}^{-n}$ is fully irreducible (and hyperbolic) [13, Theorem 5.3].

6.2. Currents. A (measured geodesic) current on $F_{k}$ is an $F_{k}$-invariant and $\sigma$-invariant positive Radon measure on $\partial^{2} F_{k}$ (refer to Section 2.6). Such measures where originally considered by Bonahon [9], see also [29]. Given a tree $T \in C V_{k}$, there is an identification between $\partial F_{k}$ and $\partial T$ used to interpret a current as a measure on the set of (bi-infinite) geodesics in $T$. Given a tight path $\alpha \subset T$, the two-sided cylinder $\operatorname{Cyl}_{T}(\alpha)$ is the collection of geodesics that contain $\alpha$; such sets determine a basis for $\partial^{2} T$, and so in turn for $\partial^{2} F_{k}$. When $\alpha$ is a fundamental domain for the action of $a \in F_{k}$ on $T^{\langle a\rangle}$, we will denote $C y l_{T}(\alpha)$ by $C y l_{T}(a)$. For a current $\nu \in \operatorname{Curr}\left(F_{k}\right)$, define $\langle a, \nu\rangle_{T}=\nu\left(C y l_{T}(a)\right)$. As $\nu$ is $F_{k}$-invariant, this is well-defined. The current is uniquely defined by the values $\langle a, \nu\rangle_{T}$. If $c \in F_{k}$ is not a proper power, then we define the counting current $\eta_{c}$ of $c$ by:

$$
\left\langle a, \eta_{c}\right\rangle_{T}=\# \text { of axes of conjugates of } c \text { in } C y l_{T}(a)
$$

If $b=c^{m}$ where $c$ is not a proper power, then $\eta_{b}=m \eta_{c}$.

Recall that an element $\phi \in$ Out $F_{k}$ is hyperbolic if it has no nontrivial periodic conjugacy classes in $F_{k}$; all such elements are necessarily non-geometric, in the sense that they are not induced by a surface homeomorphism. A hyperbolic fully irreducible element of Out $F_{k}$ acts on the projectivized space of currents $\mathbb{P C u r r}\left(F_{k}\right)$ with North-South dynamics [34]. In particular, there are both stable $\left[\mu_{+}(\phi)\right]$ and unstable $\left[\mu_{-}(\phi)\right]$ fixed projectivized currents associated to such an element. A similar statement holds for non-hyperbolic fully irreducible elements as well, after restricting to the subspace of $\mathbb{P} C u r r\left(F_{k}\right)$ consisting just of those currents in the closure of the set of counting currents of primitive elements in $F_{k}$ [31].

The support $\operatorname{Supp}(\nu)$ of a current $\nu$ is the closure of the union of all open sets $U$ such that $\nu(U)>0$. The support of a current is a lamination. The relationship between the stable currents and stable laminations of a fully irreducible element of Out $F_{k}$ is given by the proposition below. The result is probably well-known, but to our knowledge, its proof does not appear in the literature. See also [30] for closely related results. 
Proposition 6.1. Suppose $\phi \in$ Out $F_{k}$ is fully irreducible with stable and unstable laminations $\Lambda_{+}, \Lambda_{-}$and stable and unstable currents $\mu_{+}, \mu_{-}$. We have $\operatorname{Supp}\left(\mu_{ \pm}\right)=\Lambda_{ \pm}$.

Proof. Let $g: G \rightarrow G$ be a train-track representative of $\phi$. Let $a \in F_{k}$ be a primitive element and $\alpha \subset G$ the reduced loop representing its conjugacy class. Then $\alpha$ is the union of $N$ legal paths in $G$ for some $N$, so that for all $m \geq 0$, the closed path $g^{m}(\alpha)$ consists of $N$ segments of leaves of the stable lamination $\Lambda_{+}$.

The set of cylinders $C y l_{\tilde{G}}(\gamma), \gamma$ a reduced path in $\tilde{G}$, not containing any leaf of $\Lambda_{+}$give a cover of the complement of $\Lambda_{+}$. Choose one such cylinder $C y l_{\tilde{G}}(\gamma)$, so that $\gamma$ is not a subsegment of any leaf of $\Lambda_{+}$. Then for any $m \geq 0$, the reduced loop $\left[g^{m}(\alpha)\right]$ contains at most $N$ copies of the image of $\gamma$ in $G$, and hence $\eta_{\phi^{m}(a)}\left(C y l_{\tilde{G}}(\gamma)\right) \leq N$. Recall that, because $a$ was chosen to be primitive, we have the convergence of $\left[\eta_{\phi^{m}(a)}\right] \rightarrow\left[\mu_{+}\right]$. Now for a sequence $\lambda_{m}$ to give the convergence of $\frac{1}{\lambda_{m}} \eta_{\phi^{m}(a)} \rightarrow \mu_{+}$, it is necessary that $\lambda_{m} \rightarrow \infty[32$, Theorem 1.2]. Thus we have $\mu_{+}\left(C y l_{\tilde{G}}(\gamma)\right)=0$.

We have shown that $\operatorname{Supp}\left(\mu_{+}\right) \subseteq \Lambda_{+}$, a nonempty sublamination of a minimal lamination $[7,32]$. The claim of the proposition is verified.

6.3. Axes of products of Dehn twists. Let $k \geq 3$ and fix two filling cyclic trees $T_{1}, T_{2}$ with Dehn twists $\delta_{1}$ and $\delta_{2}$. Let $c_{1}, c_{2}$ denote the respective edge stabilizers. We assume that the set $\left\{c_{1}, c_{2}\right\}$ is not separable, i.e., no conjugates of $c_{1}$ and $c_{2}$ are contained in a proper free factor of $F_{k}$, nor in complementary free factors. Further, we assume that $c_{1}$ and $c_{2}$ are not simultaneously elliptic in $\overline{C V}_{k}$, i.e., $\ell_{T}\left(c_{1}\right)+$ $\ell_{T}\left(c_{2}\right) \neq 0$ for all $T \in \overline{c v}_{k}$. These conditions can be guaranteed, for instance, by requiring $c_{1}$ and $c_{2}$ to be primitive elements sufficiently far apart in the free factor complex [14].

For the remainder of this section, elements $\phi_{n} \in$ Out $F_{k}$ denote the outer automorphisms induced by the automorphisms $\delta_{1}^{n} \delta_{2}^{-n}$. For large enough $n$, the elements $\phi_{n}$ are fully irreducible and hyperbolic [13, Theorem 5.3]. From [12, Theorem 5.2], we understand the limiting behavior of the stable and unstable currents: $\left[\mu_{+}\left(\phi_{n}\right)\right] \rightarrow\left[\eta_{c_{1}}\right]$ and $\left[\mu_{-}\left(\phi_{n}\right)\right] \rightarrow\left[\eta_{c_{2}}\right]$. Using this, together with the parabolic behavior of Dehn twists on $\overline{C V}_{k}$ [15], we show that the sequence of stable and unstable trees likewise converge to the expected trees (cf., [12, Remark 5.3]).

Theorem 6.2. The trees $T_{+}\left(\phi_{n}\right) \in \overline{C V}_{k}$ converge to $T_{2}$. Similarly, the trees $T_{-}\left(\phi_{n}\right)$ converge to $T_{1}$. 
Proof. Denote $\psi_{n}=\delta_{2}^{-n} \delta_{1}^{n}$ so that $\phi_{n}=\delta_{2}^{n} \psi_{n} \delta_{2}^{-n}$. Then as the outer automorphisms are conjugate by $\delta_{2}^{n}$, we have $T_{+}\left(\phi_{n}\right)=T_{+}\left(\psi_{n}\right) \delta_{2}^{-n}$.

Recall that in $\left[12\right.$, Theorem 5.2], we determined that $\lim _{n \rightarrow \infty}\left[\mu_{-}\left(\psi_{n}\right)\right]=$ $\left[\eta_{c_{1}}\right]$. The continuity of the Kapovich-Lustig intersection form (see [32] for its definition and properties) implies that $c_{1}$ has a fixed point in an accumulation point of the sequence $\left\{T_{+}\left(\psi_{n}\right)\right\}$ (see [12, Remark 5.3]). Therefore as $c_{1}$ and $c_{2}$ are not simultaneously elliptic, $c_{2}$ has positive translation length in any such accumulation point.

As $\overline{C V}_{k}$ is compact, some subsequence of $\left\{T_{+}\left(\phi_{n}\right)\right\}$ converges. Consider such a convergent subsequence $\left\{T_{+}\left(\phi_{n_{m}}\right)\right\} \subseteq\left\{T_{+}\left(\phi_{n}\right)\right\}$. By passing to a further subsequence, we can assume that both $\left\{T_{+}\left(\phi_{n_{m_{\ell}}}\right)\right\}$ and $\left\{T_{+}\left(\psi_{n_{m_{\ell}}}\right)\right\}$ converge. Let $T_{\infty}$ denote the limit of the latter sequence. By the above remark, $c_{2}$ has positive translation length on the tree $T_{\infty}$.

Let $U \subset \overline{C V}_{k}$ be a neighborhood of $T_{2}$. As the set $\left\{T_{+}\left(\psi_{n_{m_{\ell}}}\right)\right\} \cup\left\{T_{\infty}\right\}$ is compact, and as $c_{2}$ has positive translation length on every tree therein, by [15, Theorem 13.2], there is an $N$ such that for $\ell \geq N$ we have $T_{+}\left(\phi_{n_{m_{\ell}}}\right)=T_{+}\left(\psi_{n_{m_{\ell}}}\right) \delta_{2}^{-n_{m_{\ell}}} \in U$. Therefore the subsequence $\left\{T_{+}\left(\phi_{n_{m}}\right)\right\}$ converges to $T_{2}$. As this is true for every convergent subsequence of $\left\{T_{+}\left(\phi_{n}\right)\right\}$, and as $\overline{C V}_{k}$ is compact, we have the convergence of $T_{+}\left(\phi_{n}\right) \rightarrow T_{2}$. Applying the same argument to $\phi_{n}^{-1}=\delta_{2}^{n} \delta_{1}^{-n}$ we see that $T_{-}\left(\phi_{n}\right) \rightarrow T_{1}$ as well.

Fix bases $\mathcal{T}_{1}$ and $\mathcal{T}_{2}$ for $F_{k}$, obtained from the vertex group(s) (and possibly a choice of stable letter in the case of an HNN-extension) of the Bass-Serre trees $T_{1}$ and $T_{2}$, respectively; to see how this is done, we refer to Section 3.1 of [13]. Let $T_{\mathcal{T}_{1}}$ and $T_{\mathcal{T}_{2}}$ be the Cayley trees for the basis $\mathcal{T}_{1}$ and $\mathcal{T}_{2}$, respectively. See $[12,13]$ for the details underlying these constructions.

Proposition 6.3. For sufficiently large n, we have:

$$
\tau_{c_{2}}\left(T_{\mathcal{T}_{2}}, \Lambda_{-}\left(\phi_{n}\right)\right) \geq \frac{n}{2}
$$

Proof. The proposition follows from a slight modification of the arguments from Theorem 5.2 in [12]. In its proof (equation (5.9)), we showed that for for every $\epsilon>0$ and integer $r>0$, there is an $N>0$ such that for $n \geq N$ :

$$
\frac{\left\langle c_{2}^{r}, \mu_{-}\left(\phi_{n}\right)\right\rangle_{T_{\mathcal{T}_{2}}}}{\omega_{T_{\mathcal{T}_{2}}}\left(\mu_{-}\left(\phi_{n}\right)\right)}>1-\epsilon .
$$

The $\omega_{T_{\mathcal{T}_{2}}}(\cdot)$ in the demoninator is a normalization factor whose only relevant value to the present discussion is $\omega_{T_{\mathcal{T}_{2}}}\left(\mu_{-}\left(\phi_{n}\right)\right)$; it may thus 
be treated as a positive constant. ${ }^{2}$ Equation (6.1) shows that there is a leaf of $\Lambda_{-}\left(\phi_{n}\right)=\operatorname{Supp}\left(\mu_{-}\left(\phi_{n}\right)\right)$ contained in the cylinder $C y l_{T}\left(c_{2}^{r}\right)$, and hence $\tau_{c_{2}}\left(T_{\mathcal{T}_{2}}, \Lambda_{-}\left(\phi_{n}\right)\right) \geq r$.

Following the same analysis as in [12, Theorem 5.2], fixing $r=n / 2$, one can show:

$$
\left|1-\frac{\left\langle c_{2}^{n / 2}, \mu_{-}\left(\phi_{n}\right)\right\rangle_{T_{\mathcal{T}_{2}}}}{\omega_{T_{\mathcal{T}_{2}}}\left(\mu_{-}\left(\phi_{n}\right)\right)}\right|<\left|\frac{\frac{1}{2} A n^{2}+A_{1} n+A_{2}}{A n^{2}-B_{1} n-B_{2}}\right|+\frac{\epsilon}{2}
$$

for some fixed positive constants $A, A_{1}, A_{2}, B_{1}$ and $B_{2}{ }^{3}$ Thus for large enough $n$, we have that $\left\langle c^{n / 2}, \mu_{-}\left(\phi_{n}\right)\right\rangle_{T}>0$, and hence there is a leaf of $\Lambda_{-}\left(\phi_{n}\right)=\operatorname{Supp}\left(\mu_{-}\left(\phi_{n}\right)\right)$ contained in the cylinder $C y l_{T}\left(c_{2}^{n / 2}\right)$. This implies that $\tau_{c_{2}}\left(T_{\mathcal{T}_{2}}, \Lambda_{-}\left(\phi_{n}\right)\right) \geq n / 2$, as claimed.

We can use the fact that the sequence of trees $\left\{T_{-}\left(\phi_{n}\right)\right\}$ converges to $T_{1}$ to show that the twist of $T_{-}\left(\phi_{n}\right)$ with $\Lambda_{-}\left(\phi_{n}\right)$ relative to $c_{2}$ is also approximately at least $n$.

Proposition 6.4. There exists a constant $D \geq 1$ such that for sufficiently large $n$ :

$$
\tau_{c_{2}}\left(T_{-}\left(\phi_{n}\right), \Lambda_{-}\left(\phi_{n}\right)\right) \geq \frac{n}{D}
$$

Proof. As before, let $T_{\mathcal{T}_{2}}$ be the Cayley tree corresponding to the basis $\mathcal{T}_{2}$. By Proposition 6.3, for each $n$ there is a leaf $\ell_{n}: \mathbb{R} \rightarrow T_{\mathcal{T}_{2}}$ of $\Lambda_{-}\left(\phi_{n}\right)$ that intersects the axis of $c_{2}$ in a segment of length at least $n \ell_{T}\left(c_{2}\right) / 2$. We must verify that this overlap is not significantly reduced when mapping to $T_{-}\left(\phi_{n}\right)$.

Fix an $F_{k}$-equivariant map $f: T_{\mathcal{T}_{2}} \rightarrow T_{1}$ and scale the metric on $T_{1}$ so that $\operatorname{Lip}(f) \leq 1$, and thus $B B T(f) \leq 1$ (we assume that the volume of $T_{\mathcal{T}_{2}} / F_{k}$ is 1$)$. By scaling the metrics on $T_{-}\left(\phi_{n}\right)$ we have the convergence of $T_{-}\left(\phi_{n}\right) \rightarrow T_{1}$ from Theorem 6.2. Thus for large enough $n$, we can choose equivariant maps $f_{n}: T_{\mathcal{T}_{2}} \rightarrow T_{-}\left(\phi_{n}\right)$ so that $\operatorname{Lip}\left(f_{n}\right) \leq 2$ and so $B B T\left(f_{n}\right) \leq 2$. As convergence is in the space of length functions, and as $\ell_{T_{1}}\left(c_{2}\right)>0$, there is $\delta>0$ such that $0<\delta<\ell_{T_{-}^{n}}\left(c_{2}\right)<1 / \delta$ for all $n$.

Now let $x_{n} \in \ell_{n}(\mathbb{R}) \cap T_{\mathcal{T}_{2}}^{\left\langle c_{2}\right\rangle}$ be such that $y_{n}=c_{2}^{n / 2} x_{n} \in \ell_{n}(\mathbb{R}) \cap T_{\mathcal{T}_{2}}^{\left\langle c_{2}\right\rangle}$. Thus the path $\left[f_{n}\left(x_{n}\right), f_{n}\left(y_{n}\right)\right]$ contains an arc of the axis of $c_{2}$ in $T_{-}\left(\phi_{n}\right)$ of length at least $\frac{n}{2} \ell_{T_{-}\left(\phi_{n}\right)}\left(c_{2}\right)$. Further notice that the distance from

\footnotetext{
${ }^{2}$ On the other hand, to recognize (6.1) from equation (5.9) in the proof of [12, Theorem 5.2], it should be observed that $\omega_{T_{\mathcal{T}_{2}}}\left(\eta_{c_{2}}\right)=\left\langle c_{2}^{r}, \eta_{c_{2}}\right\rangle_{T_{T_{2}}}$.

${ }^{3}$ Compare this to equation (5.9) from [12, Theorem 5.2] where the numerator of the righthand side is linear in $n$, and note that the constants $\beta_{1}, \beta_{2}$ there depend on $r$. Here in (6.2), the numerator is quadratic because of the choice of $r=n / 2$.
} 
either $f_{n}\left(x_{n}\right)$ or $f_{n}\left(y_{n}\right)$ to this arc is at most 2 (an upper bound for the bounded back tracking constant).

As $\ell_{n}: \mathbb{R} \rightarrow T_{\mathcal{T}_{2}}$ is a leaf of the unstable lamination, after tightening its image in $T_{-}\left(\phi_{n}\right)$ we obtain a geodesic $\left[f_{n}\left(\ell_{n}(\mathbb{R})\right)\right]$, and the same statement in the previous paragraph for the segment $\left[f_{n}\left(x_{n}\right), f_{n}\left(y_{n}\right)\right]$ and the axis of $c_{2}$ holds in turn for $\left[f_{n}\left(x_{n}\right), f_{n}\left(y_{n}\right)\right]$ and the geodesic $\left[f_{n}\left(\ell_{n}(\mathbb{R})\right)\right]$. Hence the leaf of $\Lambda_{-}\left(\phi_{n}\right)$ whose image in $T_{-}\left(\phi_{n}\right)$ is $\left[f_{n}\left(\ell_{n}(\mathbb{R})\right]\right.$ intersects the axis of $c_{2}$ along a segment of length at least:

$$
\begin{aligned}
\frac{n \ell_{T_{-}\left(\phi_{n}\right)}\left(c_{2}\right)}{2}-4 & >\frac{n \ell_{T_{-}\left(\phi_{n}\right)}\left(c_{2}\right)}{2}-\frac{4 \ell_{T_{-}\left(\phi_{n}\right)}\left(c_{2}\right)}{\delta} \\
& =\ell_{T_{-}\left(\phi_{n}\right)}\left(c_{2}\right)\left(\frac{n \delta-8}{4 \delta}\right) \\
& =\ell_{T_{-}\left(\phi_{n}\right)}\left(c_{2}\right) \frac{n}{D}
\end{aligned}
$$

for some constant $D>0$, provided $n>8 / \delta$. Thus

$$
\tau_{c_{2}}\left(T_{-}\left(\phi_{n}\right), \Lambda_{-}\left(\phi_{n}\right)\right) \geq \frac{n}{D}
$$

It follows from the proposition, together with Theorem 5.3, that for each element $\phi_{n}$, there is a train-track map $g_{n}: G_{n} \rightarrow G_{n}$ such that $\ell_{G_{n}}\left(c_{2}\right) \leq D^{\prime} / n$ for some constant $D^{\prime}$. Note that we are using the fact that every graph on the axis of $\phi$ represents a train-track of $\phi$.

Recall that we assumed that $\left\{c_{1}, c_{2}\right\}$ is not separable in $F_{k}$.

Lemma 6.5. If $\left\{c_{1}, c_{2}\right\}$ is not separable, then for large enough $n$, neither is $\left\{c_{2}, \delta_{1}^{n}\left(c_{2}\right)\right\}$.

Proof. This is easy to see using Whitehead graphs. Since the set $\left\{c_{1}, c_{2}\right\}$ is not separable, the union of their Whitehead graphs is connected and does not have a cut vertex (in an appropriate basis) [39]. As cancellation is bounded, for large enough $n$, the subword representing $c_{1}$ will appear as a subword of $\delta_{1}^{n}\left(c_{2}\right)$. Hence the union of the Whitehead graphs of $c_{2}$ and $\delta_{1}^{n}\left(c_{2}\right)$ will cover the union of the Whitehead graphs of $c_{1}$ and $c_{2}$. In particular, their union will be connected and will not have a cut vertex. This implies the set $\left\{c_{2}, \delta_{1}^{n}\left(c_{2}\right)\right\}$ is not separable.

Note that $\phi_{n}\left(c_{2}\right)=\delta_{1}^{n}\left(c_{2}\right)$ so that, as a consequence of the lemma, every edge in the track-track graph $G_{n}$ must be crossed by either $c_{2}$ or $\phi_{n}\left(c_{2}\right)$. Therefore, the length of $\phi_{n}\left(c_{2}\right)$ is at least $1-D^{\prime} / n=\left(n-D^{\prime}\right) / n$, and thus the Lipschitz constant for $\phi_{n}$ is at least

$$
\frac{\left(n-D^{\prime}\right) / n}{D^{\prime} / n}=n / D^{\prime}-1
$$


In particular, we have now shown that for some constant $K_{1}>0$ :

$$
\frac{1}{K_{1}} \log n \leq t r_{C V_{k}}\left(\phi_{n}\right)
$$

where $\operatorname{tr}_{C V_{k}}(\phi)=\min \left\{d_{L}(G, G \phi) \mid G \in C V_{k}\right\}$ is the minimal translation length of the element $\phi$.

We obtain the corresponding upper bound on $\operatorname{tr}_{C V_{k}}\left(\phi_{n}\right)$ by explicitly constructing a path by piecing together geodesic segments such as those constructed in Example 2.2.

As before, let $T_{\mathcal{T}_{1}}$ and $T_{\mathcal{T}_{2}}$ be the Cayley trees for the basis $\mathcal{T}_{1}$ and $\mathcal{T}_{2}$, respectively. We consider these trees as points in $C V_{k}$, with every edge of each tree having length $1 / k$. We first connect $T_{\mathcal{T}_{2}} \delta_{2}^{n}$ to $T_{\mathcal{T}_{2}}$ by a geodesic of length $\sim \log n$. Then we follow an optimal path $P$ from $T_{\mathcal{T}_{2}}$ to $T_{\mathcal{T}_{1}}$, and then connect $T_{\mathcal{T}_{1}}$ to $T_{\mathcal{T}_{1}} \delta_{1}^{n}$ with a geodesic which has length $\sim \log n$. Finally, using the $\delta_{1}^{n}$-translate $P \delta_{1}^{n}$ of $P$, we connect $T_{\mathcal{T}_{1}} \delta_{1}^{n}$ to $T_{\mathcal{T}_{2}} \delta_{1}^{n}$ (see Figure 2). As the length of $P$ is independent of $n$, translating the entire path by $\delta_{2}^{-n}$, we have for all $n$ :

$$
d_{L}\left(T_{\mathcal{T}_{2}}, T_{\mathcal{T}_{2}} \phi_{n}\right) \leq K_{2} \log n
$$

for some $K_{2}>0$.

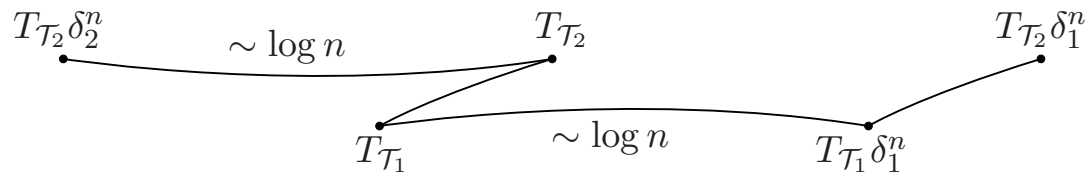

Figure 2. A path from $T_{\mathcal{T}_{2}} \delta_{2}^{n}$ to $T_{\mathcal{T}_{2}} \delta_{1}^{n}$.

Combining this upper bound with the previous lower bound, we have established the following:

Theorem 6.6. Let $T_{1}, T_{2}$ be two cyclic trees that fill with associated Dehn twist automorphisms $\delta_{1}$ and $\delta_{2}$ and let $c_{1}, c_{2}$ denote the respective edge stabilizers. Suppose that $\left\{c_{1}, c_{2}\right\}$ is not separable and that $c_{1}$ and $c_{2}$ are not simultaneouly elliptic in $\overline{C V}_{k}$. For $n \geq 1$, let $\phi_{n}$ be the outer automorphism induced by $\delta_{1}^{n} \delta_{2}^{-n}$. Then there is a constant $K=$ $K\left(T_{1}, T_{2}\right)$ such that for large enough $n$ :

(1) there is a train-track representative $g_{n}: G_{n} \rightarrow G_{n}$ such that $\ell_{G_{n}}\left(c_{2}\right) \leq K / n$, and

(2) $\frac{1}{K} \log n \leq \operatorname{tr}_{C V_{k}}\left(\phi_{n}\right) \leq K \log n$.

\section{REFERENCES}

[1] Y. Algom-KFIR, Strongly contracting geodesics in Outer space. arXiv:math/0812.1555. 
[2] Y. Algom-Kfir And M. Bestvina, Asymmetry of Outer Space. arXiv:math/0910.5408.

[3] E. Alibegović, Translation lengths in $\operatorname{Out}\left(F_{n}\right)$, Geom. Dedicata, 92 (2002), pp. 87-93. Dedicated to John Stallings on the occasion of his 65th birthday.

[4] J. Behrstock, M. Bestvina, And M. Clay, Growth rate of intersection numbers for free group automorphisms, J. Topol., 3 (2010), pp. 280-310.

[5] M. Bestvina, A Bers-like proof of the existence of train tracks for free group automorphisms. arXiv:math/1001.0325.

[6] M. Bestvina And M. Feighn, Outer limts. preprint (1992) http:// andromeda. rutgers. edu/ feighn/papers/outer.pdf.

[7] M. Bestvina, M. Feighn, And M. Handel, Laminations, trees, and irreducible automorphisms of free groups, Geom. Funct. Anal., 7 (1997), pp. 215244.

[8] M. Bestvina And M. Handel, Train tracks and automorphisms of free groups, Ann. of Math. (2), 135 (1992), pp. 1-51.

[9] F. Bonahon, Geodesic currents on negatively curved groups, in Arboreal group theory (Berkeley, CA, 1988), vol. 19 of Math. Sci. Res. Inst. Publ., Springer, New York, 1991, pp. 143-168.

[10] M. R. BRidson AND A. HAEFLIGer, Metric spaces of non-positive curvature, vol. 319 of Grundlehren der Mathematischen Wissenschaften [Fundamental Principles of Mathematical Sciences], Springer-Verlag, Berlin, 1999.

[11] M. Clay, Contractibility of deformation spaces of G-trees, Algebr. Geom. Topol., 5 (2005), pp. 1481-1503 (electronic).

[12] M. Clay and A. Pettet, Current twisting and nonsingular matrices. arXiv:math/0907.1075, to appear in Commentarii Mathematici Helvetici.

[13] — Twisting out fully irreducible automorphisms, Geom. Funct. Anal., 20 (2010), pp. 657-689.

[14] M. Clay and P. Reynolds, Stabilizers of filling free factors. in preparation.

[15] M. M. Cohen And M. Lustig, Very small group actions on R-trees and Dehn twist automorphisms, Topology, 34 (1995), pp. 575-617.

[16] T. Coulbois, A. Hilion, And M. Lustig, $\mathbb{R}$-trees and laminations for free groups. I. Algebraic laminations, J. Lond. Math. Soc. (2), 78 (2008), pp. 723736.

$[17] \longrightarrow, \mathbb{R}$-trees and laminations for free groups. II. The dual lamination of an $\mathbb{R}$-tree, J. Lond. Math. Soc. (2), 78 (2008), pp. 737-754.

$[18] \longrightarrow, \mathbb{R}$-trees and laminations for free groups. III. Currents and dual $\mathbb{R}$-tree metrics, J. Lond. Math. Soc. (2), 78 (2008), pp. 755-766.

[19] M. Culler and J. W. Morgan, Group actions on R-trees, Proc. London Math. Soc. (3), 55 (1987), pp. 571-604.

[20] M. Culler and K. Vogtmann, Moduli of graphs and automorphisms of free groups, Invent. Math., 84 (1986), pp. 91-119.

[21] B. FARB, A. Lubotzky, And Y. Minsky, Rank-1 phenomena for mapping class groups, Duke Math. J., 106 (2001), pp. 581-597.

[22] S. Francaviglia And A. Martino, The isometry group of outer space. arXiv:0912.0299.

[23] — Metric properties of outer space, Publ. Mat., 55 (2011), pp. 433-473.

[24] V. Guirardel, Cour et nombre d'intersection pour les actions de groupes sur les arbres, Ann. Sci. École Norm. Sup. (4), 38 (2005), pp. 847-888. 
[25] V. Guirardel and G. Levitt, The outer space of a free product, Proc. Lond. Math. Soc. (3), 94 (2007), pp. 695-714.

[26] U. Hamenstadt, Lines of minima in outer space. arXiv:math/0911.3620.

[27] M. Handel And L. Mosher, The expansion factors of an outer automorphism and its inverse, Trans. Amer. Math. Soc., 359 (2007), pp. 3185-3208 (electronic).

[28] — Axes in outer space, Mem. Amer. Math. Soc., 213 (2011), pp. vi+104.

[29] I. Kapovich, Currents on free groups, in Topological and asymptotic aspects of group theory, vol. 394 of Contemp. Math., Amer. Math. Soc., Providence, RI, 2006, pp. 149-176.

[30] I. Kapovich AND M. Lustig, Invariant laminations for irreducible automorphisms of free groups. arXiv:math/1104.1265.

[31] — The actions of $\operatorname{Out}\left(F_{k}\right)$ on the boundary of outer space and on the space of currents: minimal sets and equivariant incompatibility, Ergodic Theory Dynam. Systems, 27 (2007), pp. 827-847.

[32] —, Intersection form, laminations and currents on free groups, Geom. Funct. Anal., 19 (2010), pp. 1426-1467.

[33] G. Levitt and M. Lustig, Irreducible automorphisms of $F_{n}$ have northsouth dynamics on compactified outer space, J. Inst. Math. Jussieu, 2 (2003), pp. 59-72.

[34] R. Martin, Non-Uniquely Ergodic Foliations of Thin Type, Measured Currents and Automorphisms of Free Groups, PhD thesis, UCLA, 1995.

[35] H. A. Masur and Y. N. Minsky, Geometry of the complex of curves. II. Hierarchical structure, Geom. Funct. Anal., 10 (2000), pp. 902-974.

[36] Y. Minsky, Quasi-projections in teichüller space, J. Reine Angew. Math., 473 (1996), pp. 121-136.

[37] K. RAFI, A characterization of short curves of a Teichmüller geodesic, Geom. Topol., 9 (2005), pp. 179-202 (electronic).

[38] — A combinatorial model for the Teichmüller metric, Geom. Funct. Anal., 17 (2007), pp. 936-959.

[39] J. R. Stallings, Whitehead graphs on handlebodies, in Geometric group theory down under (Canberra, 1996), de Gruyter, Berlin, 1999, pp. 317-330.

Dept. of Mathematics, Allegheny College, Meadville, PA 16335

E-mail address: mclay@allegheny.edu

Dept. of Mathematics, University of British Columbia, Vancouver, BC V6T $1 \mathrm{Z2}$

E-mail address: alexandra@math.ubc.ca 